\title{
Journal of Dental
}
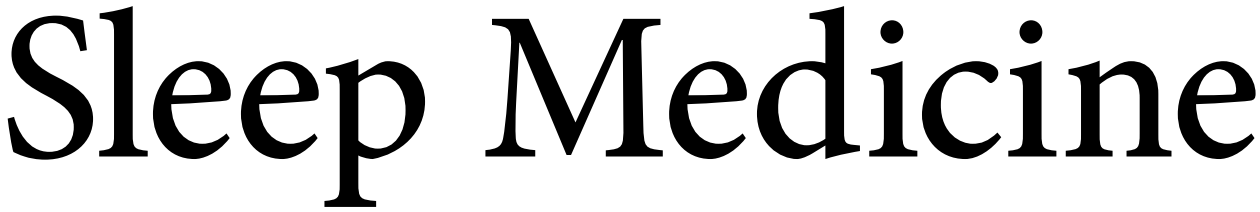

Official Publication of the American Academy of Dental Sleep Medicine | www.jdsm.org

\section{Volume 1, Number 3}

October 10, 2014

Pages 107-146

\section{In This Issue}

Oral Appliance Awareness and Beyond Dort

Connecting and Collaborating to Advance Dental Sleep Medicine

Bennett

A Novel Use of Complete Denture Prosthesis as Mandibular Advancement Device in the Treatment of Obstructive Sleep Apnea in Edentulous Subjects Tripathi, Gupta, Tripathi, Dubey

Current Barriers and Study Needs for Oral Appliance Therapy: The Personal Perspective of a Physician and Dentist Fleury, Lowe, ORal Appliance Network for Global Effectiveness Group

Looking Through the Glass Ceiling Commentary on Fleury et al.

Kuna 



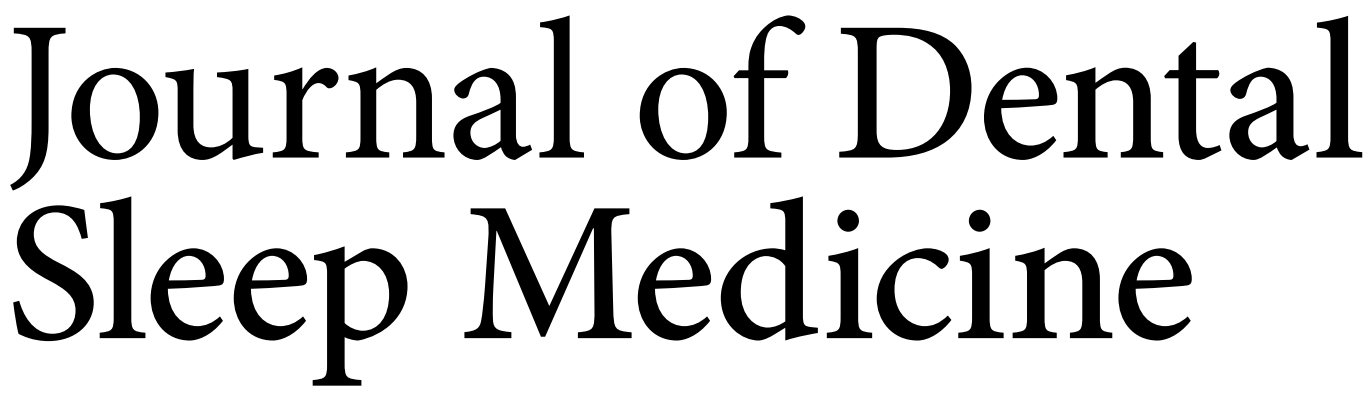

Volume 1, Number 3 | October 10, 2014 | Pages 107-146

\author{
Editor-in-Chief \\ Leslie Dort, DDS \\ Deputy Editor \\ Olivier Vanderveken, MD, PhD
}

\section{Associate Editors}

Fernanda Almeida, DDS, PhD

Gilles Lavigne, DMD, PhD

Rose Sheats, DMD

\section{Executive Director}

Jerome A. Barrett

\section{Managing Editor \\ Andrew Miller}

\author{
Editorial Board \\ Ghizlane Aarab, DDS, PhD \\ Peter Cistulli, MD, PhD \\ Greg Essick, DDS, PhD \\ Bernard Fleury, MD \\ Nelly Huynh, $\mathrm{PhD}$ \\ Sam Kuna, MD \\ Chris Lettieri, MD \\ Alan Lowe, DMD, PhD \\ Marie Marklund, DDS, $\mathrm{PhD}$ \\ Antonio Romero-Garcia, DDS, PhD \\ Satoru Tsuiki, DDS, PhD
}

Journal of Dental Sleep Medicine (JDSM) (Online 2333-9756; Website: www.jdsm.org) is published online quarterly on the $10^{\text {th }}$ of January, April, July and October by the American Academy of Dental Sleep Medicine, 2510 North Frontage Road, Darien, IL 60561-1511, phone (630) 7379705 and fax (630) 737-9790.

ANNUAL SUBSCRIPTION RATES: Subscription rates for Volume 1, 2014: Individual Online (US and International): \$40.00; Institutional Online (US and International): $\$ 70.00$. Prorated subscriptions are not available. Subscriptions begin with the January issue of the current year. Renewals should be secured as early in the year as possible to avoid uninterrupted service. Questions about subscriptions (including payments, billing procedures, or policy matters) should be directed to the AADSM office at (630) 737-9705.

ADVERTISING: Digital advertising is available on www.jdsm.org. Please contact the National Sales Account Executive at advertising@aasmnet. org for complete information.

PERMISSION TO REPRODUCE: Written permission to reproduce, in print or electronically, whole articles or any parts of works, figures or tables published in JDSM must be obtained prior to publication. Permission for republication must be arranged through the Copyright Clearance Center, Inc., 222 Rosewood Drive, Danvers, MA 01923, phone (978) 750-8400 or fax (978) 646-8600 or URL http://www.copyright.com. There are royalty fees associated with such permissions.

REPRINTS: For author reprints contact the AADSM office. For commercial reprint orders contact Cenveo Publisher Services, 4810 Williamsburg Road, \#2, Hurlock, MD 21643 or Reprints2@ cadmus.com.
DISCLAIMER: The statements and opinions contained in editorials and articles in this journal are solely those of the authors thereof and not of the American Academy of Dental Sleep Medicine, or of its officers, regents, members or employees. The Editor-in-Chief, the American Academy of Dental Sleep Medicine and its officers, regents, members and employees disclaim all responsibility for any injury to persons or property resulting from any ideas or products referred to in articles contained in this journal.

(C) 2014 American Academy of Dental Sleep Medicine 


\section{EDITORIALS}

109

Oral Appliance Awareness and Beyond

Leslie C. Dort

111

Connecting and Collaborating to Advance Dental Sleep Medicine

Kathleen Bennett

\section{ORIGINAL ARTICLES}

115

A Novel Use of Complete Denture Prosthesis as Mandibular Advancement Device in the Treatment of Obstructive Sleep Apnea in Edentulous Subjects Arvind Tripathi, Ashutosh Gupta, Suryakant Tripathi, Abhishek Dubey

\section{CASE REPORTS}

121

Oral Appliance Therapy and Atrial Fibrillation

Kathleen Bennett

\section{REVIEW ARTICLES}

123

Current Barriers and Study Needs for Oral Appliance Therapy: The Personal Perspective of a Physician and Dentist

Bernard Fleury, Alan A. Lowe, ORal Appliance Network for Global Effectiveness Group

\section{COMMENTARY}

\section{9}

\section{Looking Through the Glass Ceiling}

Commentary on Fleury B, Lowe AA, ORal Appliance Network for Global Effectiveness Group. Current barriers and study needs for oral appliance therapy: the personal perspective of a physician and dentist. Journal of Dental Sleep Medicine 2014;1(3):123-127.

Samuel T. Kuna

\section{SPECIAL ARTICLES}

\section{1}

Rapid Maxillary Expansion for Pediatric Sleep Disordered Breathing

Rose D. Sheats

\section{LETTERS TO THE EDITOR}

\section{5}

A Little Insurance Information That Might Keep You Out of Harm's Way

David B. Schwartz

137

What's in a Name?

Todd D. Morgan

\section{NEWS AND UPDATES}

\section{9}

Generating Awareness for Oral Appliance Therapy L.C. Williams \& Associates

141

\section{AADSM News and Updates} AADSM Staff

\section{3}

Biographical Information for the 2014-2015 American Academy of Dental Sleep Medicine Board of Directors

Leslie C. Dort

\section{5}

Membership Survey Summary

Leslie C. Dort 


\title{
Oral Appliance Awareness and Beyond
}

\author{
Leslie C. Dort, DDS, Diplomate, ABDSM, Editor-in-Chief Journal of Dental Sleep Medicine
}

Calgary, Alberta, Canada

This issue of the Journal of Dental Sleep Medicine (JDSM) 1 contains a summary of the AADSM public awareness campaign now beginning its third year. A pre-campaign survey revealed that although the public was aware of OSA as a common disease it was unaware that an oral appliance provided by a dentist is possible treatment. The article "Generating Awareness for Oral Appliance Therapy" reviews for AADSM members some of activities conducted to date and the plan for future activities. ${ }^{1}$ An important component of the campaign is the members “Toolkit." The "Toolkit" contains documents and action steps to create public awareness of oral appliance therapy in your community and suggestions for customizing these items for your particular circumstances.

The summary includes a tip on one way to begin using the "Toolkit." All members should find this a relatively easy way to begin. Members will find the full "Toolkit" in the member's section of aadsm.org.

This campaign to raise awareness of oral appliance therapy should be considered just part of the public education that we can provide. Dentists with education in sleep should consider themselves "first responders" in the area of public education in sleep.

Some current societal views of sleep have led to sleep habits that are detrimental to both individual and societal health, and safety.

Education of patients, colleagues and other in our sphere of influence should be considered an important role for those of us broadly knowledgeable in the field of sleep. Patients, colleagues, family and community groups can all benefit from education not only on the role oral appliance therapy but beyond into basic sleep hygiene.

When and how much to sleep, avoiding shift work and circadian disruption when possible and providing better support to those essential services that must include shift work: we can pass on core information on these topics.
The recent American Pediatric Association policy statement on "School Start Times for Adolescents" raises an issue to be addressed in all communities. ${ }^{2}$ School start times before 8:30 am contribute to insufficient sleep in adolescents. Insufficient sleep significantly affects the health, safety and academic performance in adolescent. Class times based on teacher/parent/local government preferences rather than the healthiest time for adolescents may have long-range negative impacts on society.

Dental sleep medicine practitioners are ideally placed to help society "relearn" best sleep practices.

Look for future issues of JDSM to have more information on sleep and health.

\section{CITATION}

Dort LC. Oral appliance awareness and beyond. Journal of Dental Sleep Medicine 2014;1(3):109.

\section{REFERENCES}

1. L.C. Williams \& Associates. Generating awareness for oral appliance therapy. Journal of Dental Sleep Medicine 2014;1:139-40.

2. American Pediatric Association. School start times for adolescents. Pediatrics 2014;134:642-9.

\section{SUBMISSION \& CORRESPONDENCE INFORMATION}

Submitted for publication September, 2014

Accepted for publication September, 2014

Address correspondence to: Leslie C. Dort, DDS, 1016-68th Ave SW, Suite 150, Calgary, AB T2V 4J2, Canada; Tel: (403) 202-4905; Fax: (403)202-

0266; E-mail: lcdort@gmail.com

\section{DISCLOSURE STATEMENT}

Dr. Dort is Editor-in-Chief of Journal of Dental Sleep Medicine. 



\title{
Connecting and Collaborating to Advance Dental Sleep Medicine
}

\author{
Kathleen Bennett, DDS, Diplomate, ABDSM
}

Kathleen M. Bennett, DDS, LLC, Cincinnati, OH; President, American Academy of Dental Sleep Medicine

W e live in an exciting time to be a dentist who provides oral appliance therapy (OAT) for obstructive sleep apnea (OSA), and the American Academy of Dental Sleep Medicine (AADSM) is in the midst of a strategic stage in its growth and development as the leading professional society for dentists who treat sleep-disordered breathing.

More people than ever before are in need of our expertise and care. Data from the Wisconsin Sleep Cohort suggest that the prevalence of OSA has increased substantially over the last two decades, likely due to the widespread obesity epidemic in the U.S. ${ }^{1}$ The most current prevalence estimates are that 26 percent of adults between 30 and 70 years of age have at least mild OSA, and 10 percent of adults have moderate or severe disease. Based on this data, it has been estimated that OSA now afflicts at least 25 million adults in the U.S. ${ }^{2}$

Addressing this growing burden of OSA, we now have more dentists than ever before who are practicing dental sleep medicine. In the span of about two-and-a-half decades, the AADSM has grown from a small group to an expansive fellowship of colleagues, with a membership of more than 3,000 individuals. More dentists in more places throughout the U.S. and abroad are offering OAT, a treatment that is life-changing for many and that has the potential to be life-saving. ${ }^{3}$

Although we celebrate the rapid and necessary growth of dental sleep medicine, and recognize the contributions of those leaders who paved the way for us, we also must recognize the challenges that are both before us and on the horizon. Currently, the AADSM Board of Directors is in the initial stages of a strategic planning process that will address the most pressing concerns facing our organization and our membership. As we embark on this journey in the first half of my tenure as 20142016 president of the AADSM, I anticipate that we will spend much of our time considering and discussing competition, connection and collaboration.

\section{COMPETITION}

Dentists are no strangers to competition. In fact, I think we thrive on it. Most general dentists practice in markets that are highly competitive, where patients have numerous options available for their dental care. To succeed we learn to be proactive and innovative while understanding that our best form of advertising is to provide the highest quality of care for our patients. $^{4}$

Dental sleep medicine was founded on this entrepreneurial spirit, which led to a veritable explosion of innovation and invention in the field. ${ }^{5}$ Currently, there are 107 anti-snoring devices and 23 jaw repositioning devices for snoring and OSA that have received $510(\mathrm{k})$ clearance for commercial distribution from the U.S. Food and Drug Administration. ${ }^{6}$ However, in the years ahead it is likely that continued competition driven by basic market forces will lead to contraction in the number of oral appliances available to clinicians.

We also are entering an era in which we will see increased competition with other therapies for OSA. Until recently OAT has enjoyed a certain degree of exclusivity as the non-invasive alternative to continuous positive airway pressure (CPAP) therapy, a position that is undergirded by a robust body of research and evidence-based clinical standards developed jointly by the AADSM and the American Academy of Sleep Medicine (AASM). However, in the last few years new technology and novel approaches to treatment have expanded the therapeutic options for OSA. These treatments include singleuse expiratory positive airway pressure (EPAP) valves inserted into each nostril and sealed with adhesive (e.g., Provent); oral pressure therapy (OPT), which stabilizes the airway using continuous negative pressure created by a vacuum pump connected with tubing to a polymer mouthpiece (e.g., Winx); a neck-worn device that delivers vibro-tactile feedback to limit supine sleep and reduce positional OSA (e.g., Night Shift); and an upper airway stimulation (UAS) device that requires surgical implantation but is considered to be less invasive than traditional surgical approaches (e.g., Inspire).$^{7-10}$

Because of the increasing diversity of treatment options, it is critical that we refine our methods to identify the candidates who will be most responsive to OAT. Dental sleep medicine has never involved a "one size fits all" approach to treatment. Now more than ever it is incumbent on us to develop validated protocols to predict treatment response, which will help us thrive in a more competitive treatment environment. ${ }^{11}$

\section{CONNECTION}

Although the AADSM membership has expanded significantly since its establishment in 1991, there remains much more room for growth. According to the Bureau of Labor Statistics of the U.S. Department of Labor, dentists held about 146,800 jobs in 2012. ${ }^{12}$ Over the next decade, dentistry is expected to grow 16 percent, which will be faster than the average of all occupations, producing more than 23,000 additional jobs by 2022 .

Because of the high prevalence and destructive health consequences of OSA, we need many more dentists to join us in reducing the burden of snoring and sleep apnea. The recent cover story of AGD Impact is the latest evidence that the dental community is becoming more aware of dental sleep medicine, and the AADSM will continue to be the premier professional connection for dentists interested in treating sleep-disordered breathing. ${ }^{13}$

The AADSM also will continue to connect dentists to an increased offering of professional education opportunities that promote excellence in dental sleep medicine. Our diverse portfolio of courses-essentials, advanced, practical demonstration, 
and board review-provides learning opportunities for dentists at every level of experience, and our forthcoming online learning modules will greatly enhance the accessibility of this education. Each year the AADSM annual meeting also is the foremost educational event in dental sleep medicine, allowing dentists to learn from leaders in the field while connecting with colleagues from across the country. I encourage you to make plans to join us for the AADSM $24^{\text {th }}$ annual meeting, which will be held June 4-6, 2015, in Seattle, Washington. The knowledge gained from this education promotes clinical excellence, and the AADSM Dental Sleep Medicine Facility Accreditation program gives dentists the opportunity to distinguish their practice by demonstrating that they meet high standards of proficiency, professionalism and quality patient care.

Through an ongoing national public relations campaign, the AADSM also continues to connect with consumers, building awareness of the effectiveness and benefits of oral appliance therapy. In two years the campaign has generated more than 2,000 stories and 125 million media impressions in both local publications and high-profile, national magazines such as Woman's Day and Real Simple. This media coverage has raised the profile of dental sleep medicine and continues to drive patients to the online directory of member dentists on the AADSM website.

\section{COLLABORATION}

As dentists we have a tendency to be individualists, private practice pioneers who start our own business and enjoy working independently. Therefore, to the dentist who is a novice in providing oral appliance therapy for OSA, the required collaboration with a physician can seem counterintuitive. Yet this collaboration, which is outlined in the AADSM treatment protocol, is essential: It ensures professional alignment with state laws governing the practice of medicine and dentistry, while promoting effective, long-term care for patients with OSA. ${ }^{14}$ Building on the foundation of our treatment protocol, the AADSM Board of Directors recently approved the planning and implementation of a consensus conference to develop a comprehensive standard of care paper for the practice of dental sleep medicine. We believe that this will be a definitive resource, delineating the role of the dentist in the collaborative care of patients with OSA.

Since its establishment in 1991, the AADSM has been intentional at developing a positive working relationship with the AASM and our sleep medicine physician colleagues. Our Board of Directors purposefully emphasized this important dynamic in the language of the AADSM vision statement, "To lead and promote dentistry's role in collaboration with our physician colleagues in reducing the burden of snoring and sleep apnea." Together our organizations developed a joint policy statement to clarify the boundaries of this collaboration at a time when the lines are being blurred by companies that are marketing diagnostic devices and home sleep testing services directly to dentists. ${ }^{15}$ Currently, we are working together to update the practice parameters for oral appliance therapy, which brought unprecedented recognition to dental sleep medicine when it was published in $2006 .{ }^{16}$ Furthermore, AADSM leaders will be presenting a lecture about oral appliance therapy to an audience of 400 sleep medicine physicians at Sleep Medicine Trends 2015 in February.
Our constructive collaboration with physicians is especially important due to the ongoing implementation of the Patient Protection and Affordable Care Act (ACA), which is emphasizing integrated, coordinated health care. It will be critical for dentists who provide oral appliance therapy to be a "good neighbor" to the patient-centered medical home, working closely with primary care providers and sleep specialists to provide seamless, value-based care for patients with OSA. ${ }^{17}$ For some dentists the road less travelled may be to provide oral appliance therapy "under one roof" as part of a sleep medicine practice, allowing for direct interaction with physicians and promoting streamlined patient care. ${ }^{18}$

\section{CONCLUSION}

During this period of transition when the U.S. health care system is changing dramatically, it is more important than ever for the AADSM to define the field of dental sleep medicine and solidify the role of the dentist in the treatment of snoring and sleep apnea. Over the next two years the AADSM will focus on issues of broad importance to the field: setting standards of care, outlining a pathway to board certification, developing outcome measures, promoting comparative effectiveness research, and preparing members for changes in insurance reimbursement. The AADSM also will increase its health policy engagement by representing the interests of members, establishing relationships with insurers, and helping members understand the impact of the ACA on the practice of dental sleep medicine.

In the years ahead the professional path of every dentist who provides OAT is sure to take some twists and turns as the U.S. health care system evolves, oral appliance innovations are introduced, and new OSA treatment options spark increased competition. However, the AADSM will continue to help members make the connections and promote the collaborations necessary to navigate these changes successfully.

\section{CITATION}

Bennett K. Connecting and collaborating to advance dental sleep medicine. Journal of Dental Sleep Medicine 2014;1(3):111-113.

\section{REFERENCES}

1. Peppard PE, Young T, Barnet JH, et al. Increased prevalence of sleepdisordered breathing in adults. Am J Epidemiol 2013;177:1006-14.

2. National Healthy Sleep Awareness Project. Stop the snore: sleep apnea action urgent for those at risk. 2014 Aug 12 [cited 2014 Sept 2]. Available from: http://sleepeducation.org/docs/default-document-library/stopthe-snore-sleep-apnea-action-urgent-for-those-at-risk.pdf.

3. Anandam A, Patil M, Akinnusi M, et al. Cardiovascular mortality in obstructive sleep apnoea treated with continuous positive airway pressure or oral appliance: an observational study. Respirology 2013;18:1184-90.

4. Dort LC. Quality. Journal of Dental Sleep Medicine 2014;1:81.

5. Rogers RR, Remmers J, Lowe AA, Cistulli PA, Prinsell J, Pantino D, Rogers MB. History of dental sleep medicine. Journal of Dental Sleep Medicine 2014;1:67-74.

6. U.S. Food and Drug Administration. 510(k) premarket notification. Search database. Updated 2014 Aug 25 [cited 2014 Aug 29]. Available from: http://www.accessdata.fda.gov/scripts/cdrh/cfdocs/cfpmn/pmn. $\mathrm{cfm}$.

7. Kryger MH, Berry RB, Massie CA. Long-term use of a nasal expiratory positive airway pressure (EPAP) device as a treatment for obstructive sleep apnea (OSA). J Clin Sleep Med 2011;7:449-53. 
8. Colrain IM, Black J, Siegel LC, et al. A multicenter evaluation of oral pressure therapy for the treatment of obstructive sleep apnea. Sleep Med 2013;14:830-7.

9. Levendowski DJ, Seagraves S, Popovic D, Westbrook PR. Assessment of a neck-based treatment and monitoring device for positional obstructive sleep apnea. J Clin Sleep Med 2014;10:863-71.

10. Strollo PJ Jr, Soose RJ, Maurer JT, et al. Upper-airway stimulation for obstructive sleep apnea. N Engl J Med 2014;370:139-49.

11. Scherr SC, Dort LC, Almeida FR, et al. Definition of an effective oral appliance for the treatment of obstructive sleep apnea and snoring: a report of the American Academy of Dental Sleep Medicine. Journal of Dental Sleep Medicine 2014;1:39-50.

12. Bureau of Labor Statistics, U.S. Department of Labor. Occupational outlook handbook. Dentists. 2014-15 Edition [cited 2014 Aug 28]. Available from: http://www.bls.gov/ooh/healthcare/dentists.htm.

13. Shepherd L. Breathing easier: helping patients with obstructive sleep apnea. AGD Impact 2014;42:16-20.

14. American Academy of Dental Sleep Medicine. AADSM treatment protocol: oral appliance therapy for sleep disordered breathing: an update for 2013. June 2013 [cited 2014 Sept. 2]. Available from http:// www.aadsm.org/treatmentprotocol.aspx.

15. American Academy of Sleep Medicine and American Academy of Dental Sleep Medicine. Policy statement on the diagnosis and treatment of obstructive sleep apnea. 2012 Dec 7 [cited 2014 Sept 2]. Available from: http://www.aadsm.org/resources/pdf/jointpolicy.pdf.
16. Kushida CA, Morgenthaler TI, Littner MR, et al. Practice parameters for the treatment of snoring and obstructive sleep apnea with oral appliances: an update for 2005. Sleep 2006;29:240-3.

17. Morgenthaler TI, Badr MS. Ensuring patient access to sleep specialty care in the evolving U.S. healthcare system: introducing the Welltrinsic Sleep Network. An investment of the American Academy of Sleep Medicine. J Clin Sleep Med 2014;10:463-4.

18. Sharma S, Essick G, Schwartz D, Aronsky AJ. Sleep medicine care under one roof: a proposed model for integrating dentistry and medicine. J Clin Sleep Med 2013;9:827-33.

\section{SUBMISSION \& CORRESPONDENCE INFORMATION}

Submitted for publication September, 2014

Accepted for publication September, 2014

Address correspondence to: Kathleen Bennett, DDS, Diplomate, ABDSM, 222 Piedmont Avenue, Suite 8300, Cincinnati, OH 45219; Tel: (513) 871-

9111; Fax: (513) 467-0943; E-mail: kmbennettdds@gmail.com

\section{DISCLOSURE STATEMENT}

Dr. Bennett has indicated no financial conflicts of interest. 



\title{
A Novel Use of Complete Denture Prosthesis as Mandibular Advancement Device in the Treatment of Obstructive Sleep Apnea in Edentulous Subjects
}

\author{
Arvind Tripathi, MDS, FACS ${ }^{1}$; Ashutosh Gupta, BDS ${ }^{1}$; Suryakant Tripathi, MD, FCCP²; Abhishek Dubey, MSc ${ }^{3}$ \\ ${ }^{1}$ Saraswati Dental College and Hospital, Lucknow, India; Saraswati Dental College and Hospital, Lucknow; ${ }^{2}$ Department of Pulmonary Medicine, \\ King George's Medical University, Lucknow, India; ${ }^{3} \mathrm{PhD}$ Scholar at King George's Medical University, Lucknow, India
}

\begin{abstract}
STUdy ОвJеCTIVE: Mandibular advancement devices have been used to efficiently treat mild to moderate obstructive sleep apnea by effecting a forward positioning of the tongue- mandible complex and a resultant increase in oropharyngeal volume. These devices are essentially tooth anchored and hence, work well in dentate patients. However, it is difficult to anchor such devices in an edentate mouth. This clinical study presents a case series on use of complete dentures modified to function as mandibular advancement device.

MethoDs: Ten edentulous OSA patients (8 male and 2 female, aged $61 \pm 4$ years; BMI $22 \pm 5$; AHI 15-30) who volunteered and provided written informed consent were included in the study. All of them were provided with complete dentures, and after 1 month of comfortable wear and attaining optimum function, the prosthesis was modified to serve alternatively as a mandibular advancement device. Use of complete dentures as MAD was recorded separately. Five variables were assessed preoperatively and after 6 months of wearing the modified mandibular advancement device. The variables were: sleep efficiency, apnea-hypopnea index, oxygen desaturation events/h, mean $\mathrm{O}_{2}$ saturation, and snoring index. Overnight polysomnography was done to evaluate the sleep variables preoperatively and after 6 months of wearing the modified mandibular advancement device.
\end{abstract}

RESULTS: A significant decrease in apnea-hypopnea index (AHI) from 22.5 preoperatively to 4.8 postoperatively $(\mathrm{p}=0.005)$ and snoring index from 7.9 preoperatively to 2.1 postoperatively $(p=0.005)$ was observed after 6 months of use of the modified oral appliance. Sleep efficiency increased from $62.55 \%$ preoperatively to $73.20 \%$ postoperatively $(\mathrm{p}=0.005)$.

ConcLusions: The results indicate that within the limits of the present study, this specific MAD can be effective in reducing sleep apnea by lowering the AHI, reducing snoring and oxygen desaturation events/h and increasing sleep efficiency.

KEYWORDS: mandibular advancement device, obstructive sleep apnea, complete edentulism

Citation: Tripathi A, Gupta A, Tripathi S, Dubey A. A novel use of complete denture prosthesis as mandibular advancement device in the treatment of obstructive sleep apnea in edentulous subjects. Journal of Dental Sleep Medicine 2014;1(3):115-119.

$\mathrm{O}$ bstructive sleep apnea (OSA) syndrome is a serious medical condition that affects substantial number of individuals. The worldwide OSA prevalence rates in adults ranges between $3.5 \%$ to $27 \% .^{1,2}$ In India, its prevalence ranges between $3 \%$ and $28 \%$ in men and $2.2 \%$ and $16 \%$ in women. ${ }^{3-5}$ It is characterized by a disruption of normal sleep architecture due to partial or complete obstruction of the respiratory airflow. Airflow obstruction leads to a reduction of blood oxygen saturation, also known as hypoxemia. This produces an arousal in an attempt to reopen the airway. Recurrent arousals disrupt the sleep architecture, diminish the quality of sleep, and cause excessive daytime somnolence. These features have a deleterious effect on the quality of life and state of health of affected individuals. ${ }^{6}$

Studies suggest that edentulism worsens sleep apnea. ${ }^{7-9}$ Loss of vertical dimension of occlusion causes reduction of the lower facial height and rotation of the mandible. Sleeping without dentures may be associated with a significant increase in the AHI. ${ }^{7}$ The lack of a vertical stop facilitates an overclosure of the maxillomandibular system and an upper airway collapse. Even in a patient using complete dentures, the vertical dimension of occlusion worsens continuously because of bone resorption. ${ }^{2}$

During the last two decades, there has been increased interest in oral appliance $(\mathrm{OA})$ as a treatment modality for OSA. The $\mathrm{OA}$ is used during night; it protrudes the mandible and thereby opens the airway. The oral appliance is easy to use as it does not require electrical power and does not make any noise; crossover studies have shown that patients prefer OA therapy to CPAP, which leads to good compliance.

Oral appliances can either be prefabricated or custom made. Custom-made mandibular advancement devices (MADs) are fabricated on dental casts of the patient. All MADs have been developed for dentate patients in whom the remaining dentition provides anchorage and stability to the device. Only tongue retaining devices (TRD) have been recommended for edentate patients. ${ }^{10-12}$ Earlier clinical studies on the use of dentures for the treatment of OSA ${ }^{13-15}$ have not reported data on mandibular advancement. This clinical study presents a case series on use of complete dentures modified to function as MAD.

\section{METHODS}

The present study was conducted in the Department of Prosthodontics, Saraswati Dental College \& Hospital, Lucknow, in collaboration with Department of Pulmonary Medicine, King George's Medical University, Lucknow. Prior approval of the Institutional ethical committee had been obtained.

Ten edentulous OSA patients ( 8 male, 2 female, age $61 \pm 4$ years; BMI $22 \pm 5$; AHI 15-30), who volunteered and provided written informed consent were included in the study. A provisional selection of patients was based on subjective evaluation of symptoms, according to the Epworth Sleepiness Scale and the 


\section{Figure 1-The snoring index}

Evaluation of snoring as reported by bed partner (circle one):

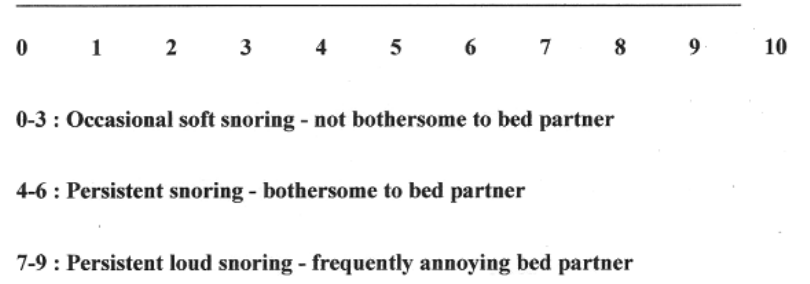

10 : Heroic snoring - continuous, loud snoring not tolerated by bed partner

Berlin Questionnaire. Such patients were subjected to overnight polysomnography to confirm their actual status before being included as study subjects. Five variables were assessed preoperatively and after 6 months of wearing the modified MAD. These variables were: sleep efficiency, AHI, oxygen desaturation events/h, mean $\mathrm{O}_{2}$ saturation, and snoring index.

For recording sleep efficiency, AHI, oxygen desaturation events/h, limb movement and mean $\mathrm{O}_{2}$ saturation, overnight polysomnography was performed twice (preoperatively and after 6 months of wearing the appliance).

Overnight polysomnography sleep study (S-7000, Cogent technologies, EMBLA System Inc) included electroencephalograms (EEG); (C3-A2, C4-A1, O2-A1, O3-A2), bilateral electrooculogram (ROC, LOC), chin and leg electromyogram (EMG), nasal airflow, thoracic and abdominal movements, electrocardiogram (ECG), $\mathrm{O}_{2}$ saturation measurement by finger pulse oximeter, and body position recorders. Apnea-hypopnea index (AHI) was calculated with the help of Somnologica Studio software. The apnea episodes were defined as complete cessation of airflow $\geq 10 \mathrm{~s}$, and hypopnea was defined as $\geq 50 \%$ reduction in oronasal airflow accompanied by a reduction of $\geq 4 \%$ oxygen saturation calculated by pulse oximetry. AHI was determined by the frequency of these events per hour during sleep time based on the results of the overnight polysomnography. Recorded polysomnographic data were cross checked manually for scoring of sleep stages, apneas, and hypopnea events.

For grading of snoring intensity, the bed partner snoring evaluation index tool was used (Figure 1). As the sample size was small a nonparametric assessment plan was adopted. Data were analyzed using SPSS Version 15.0. Wilcoxon signed rank test was used to evaluate before-after changes. A p value $<0.05$ indicated statistically significant difference.

\section{Complete Denture Prosthesis Modified To Function as a Mandibular Advancement Device}

Maxillary and mandibular complete dentures were fabricated by the conventional standard procedure. With the maxillary denture in the patient's mouth, facebow records were taken and transferred onto the Hanau "wide vue" articulator. A 3-mm thick wax interocclusal record was made and maximum protrusive jaw movement was done, as dictated by the condylar guidance path. The mandibular denture was mounted on the articulator using the wax record. The wax record was
Figure 2-Maxillary denture with hole at $1^{\text {st }}$ molar region

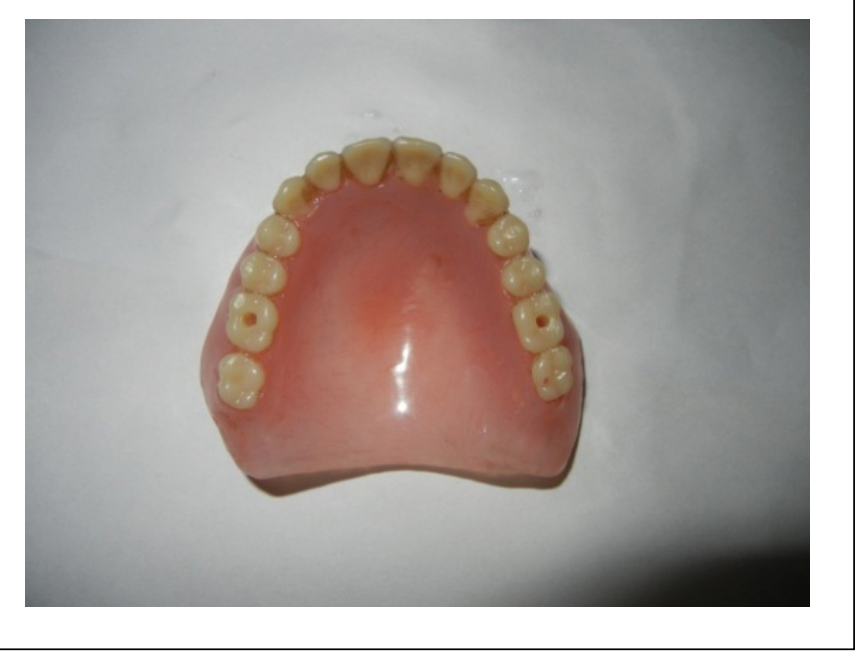

removed and the centric lock of the articulator was fixed at $70 \%$ of maximum protrusion. ${ }^{16} \mathrm{Next}$, the heat cure soft liner (Permasoft) was added on the occlusal surface from the first premolar up to the second molar. The articulator was closed and the soft liner was allowed to set. The soft liner wafer was then cured, and thereafter the entire assembly (the maxillary and mandibular dentures and the soft liner wafer) was removed from the articulator. A hole was drilled at the first molar region on the intaglio surface of maxillary denture bilaterally, passing through the soft liner wafer up to the occlusal surface of mandibular denture, such that the two could be engaged securely. The soft liner wafer was fixed to the maxillary and mandibular denture using brass screws passing through the hole. This was done so that dentures could provide the function of mastication by removing the wafer during the daytime and then function as a mandibular advancement prosthesis by placing the wafer and tightening it with screws during sleep at night. The wafer engaged the posterior occlusion and created an interarch anterior space, which facilitated unobstructed inspiration even during sleep. The 3-piece modified mandibular advancement device (maxillary and mandibular dentures with soft liner wafer in between), was inserted and adjusted intraorally. The patient was provided with a brass mini-screwdriver and was instructed on how to screw the denture and soft liner wafer during night and to unscrew it during daytime (Figures 2-8).

\section{RESULTS}

A significant decrease in AHI from 22.5 preoperatively to 4.8 postoperatively $(78.67 \%)$, snoring index (from 7.9 preoperatively to 2.1 postoperatively [73.42\%]) and oxygen desaturation events/h (from 18.84 preoperatively to 5.04 postoperatively) was observed after 6 months of wearing the modified oral appliance. Sleep efficiency increased from $62.55 \%$ preoperatively to $73.20 \%$ postoperatively. However, there was no statistically significant improvement in mean $\mathrm{O}_{2}$ saturation event ( $\mathrm{p}$ value $>0.05$; Table 1). For a mean change of 10.65 units with a pooled standard deviation of 6.49 units, the power of test was $100 \%$. 
Figure 3-Corresponding hole in the mandibular denture

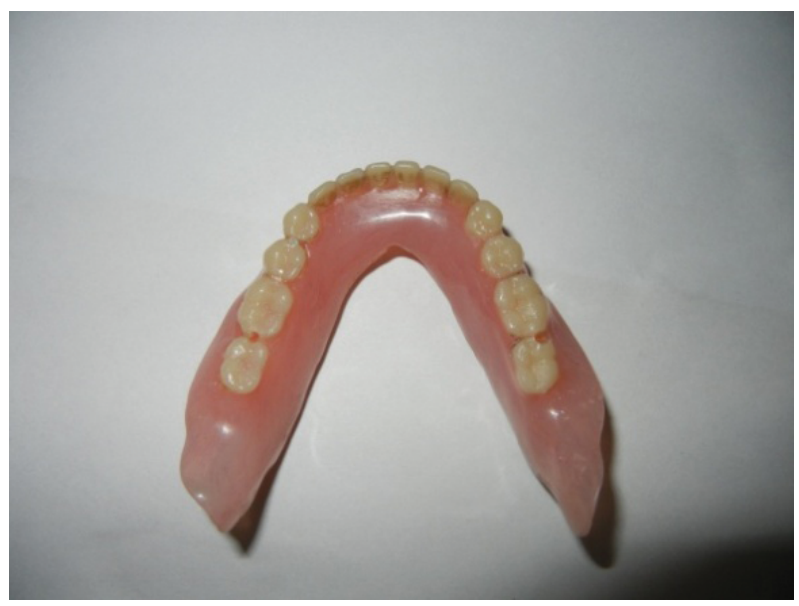

Figure 5-The screw used to immobilize the denture and the soft liner wafer during night.

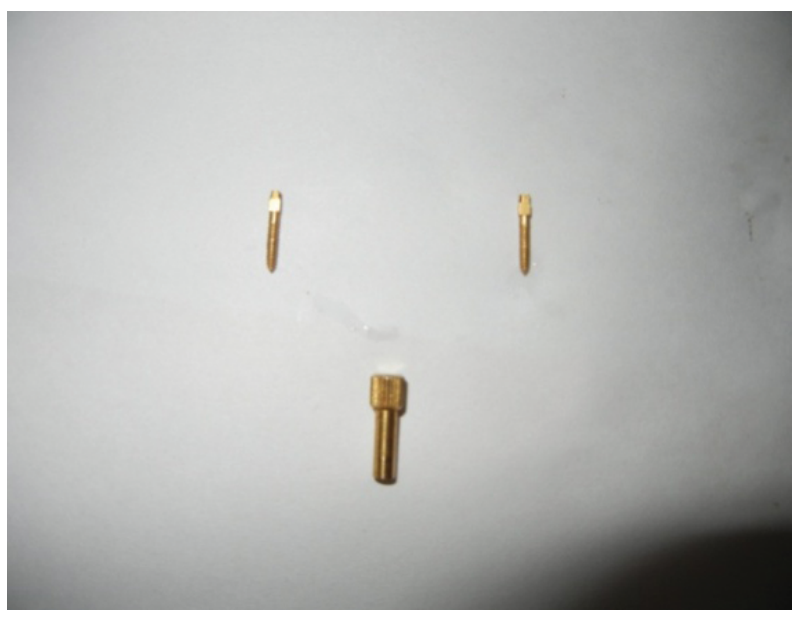

Figure 7-The 3-unit modified MAD appliance.

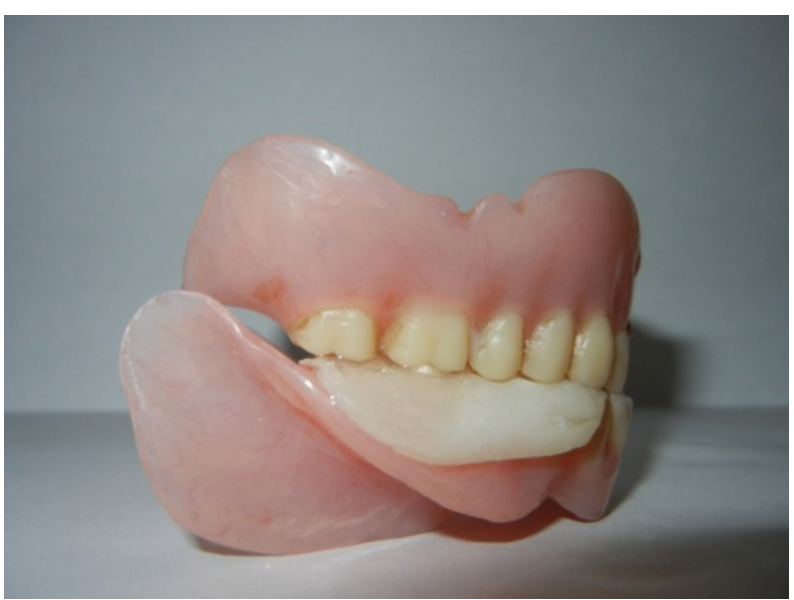

Figure 4-Mandibular denture with soft liner wafer

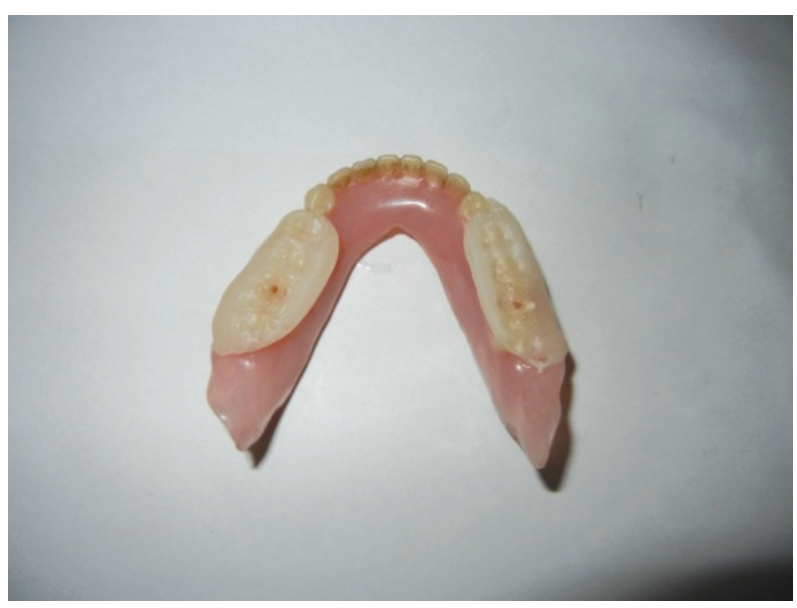

Figure 6-Intanglio surface of maxillary denture showing the position of screw

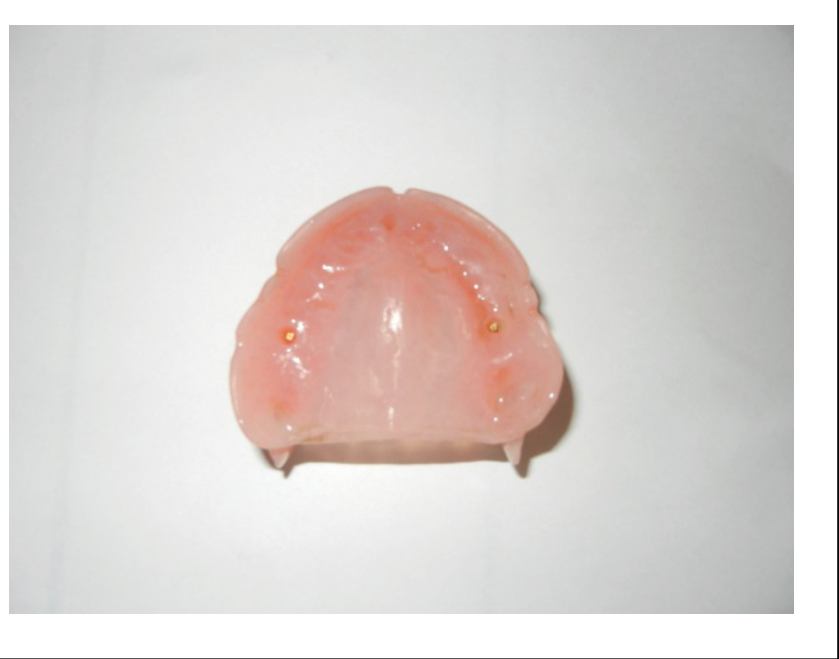

Figure 8-The intraoral view of modified mandibular advancement device.

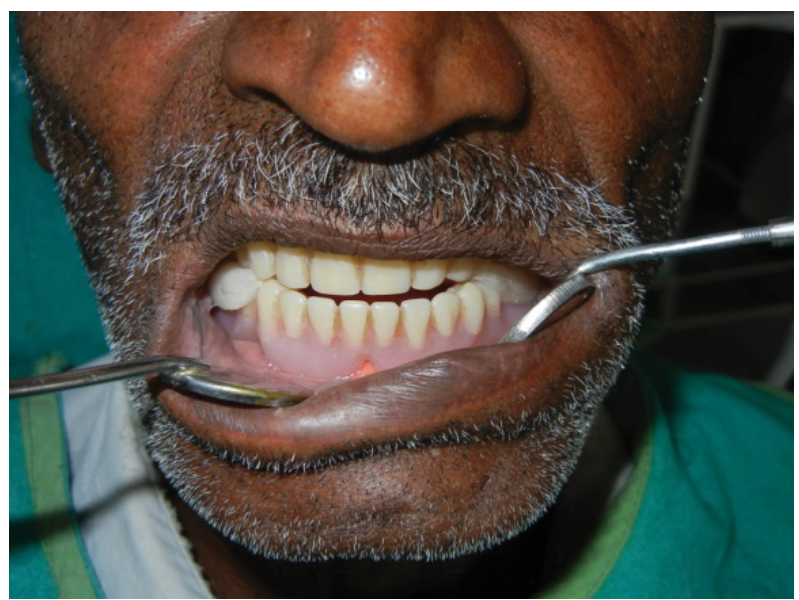


Table 1-Statistical analysis

Parameter
Sleep efficiency (\%)
AHI
Oxygen desaturation events/h
Mean $\mathrm{O}_{2}$ saturation
Snoring index

\begin{tabular}{rr}
\multicolumn{2}{c}{ Before } \\
\hline Mean & \multicolumn{1}{c}{ SD } \\
62.55 & 5.46 \\
22.50 & 5.19 \\
18.84 & 19.23 \\
93.43 & 1.14 \\
7.90 & 1.37
\end{tabular}

\begin{tabular}{rcc}
\multicolumn{3}{c}{ Change } \\
\hline Mean & SD & \% Change \\
10.65 & 6.49 & 17.03 \\
-17.70 & 3.33 & -78.67 \\
-13.80 & 17.28 & -73.25 \\
0.62 & 1.15 & 0.66 \\
-5.80 & 1.14 & -73.42
\end{tabular}

\begin{tabular}{cc}
\multicolumn{2}{c}{$\begin{array}{c}\text { Significance of } \\
\text { change (Wilcoxon } \\
\text { signed rank test) }\end{array}$} \\
\hline $\mathbf{Z}$ & $\mathbf{p}$ \\
2.803 & 0.005 \\
2.809 & 0.005 \\
2.803 & 0.005 \\
1.682 & 0.093 \\
2.831 & 0.005
\end{tabular}

\section{DISCUSSION}

The present study found the use of complete dentures with modifications to be an effective mandibular advancement device for the treatment of obstructive sleep apnea in a sample of edentulous patients. The MAD was retained by the edentulous arches and stabilized by screwing on the interocclusal wafer with the maxillary and the mandibular dentures, thus rendering the three as one composite unit. Placement of the wafer between the dentures increased the interarch distance (vertically) enough to make the MAD appliance self-retentive even at rest. Since the MAD was a mere modified version of dentures already in use with no discomfort, no fresh complaint of soreness or ulceration was reported. The MAD was prepared at $70 \%$ of the maximum mandibular protrusion recorded, to avoid any possible anterior impingement of the glenoid fossae by the condyles as done by Keyf et al. ${ }^{16}$ Since the appliance was an altered form of a well-adjusted dental prosthesis, the patients did not report any discomfort. However, in certain cases, altered maxillomandibular relationship (due to mandibular advancement) may lead to impingement of dental prosthesis in newer areas and cause fresh denture soreness. In this event, such spots must be identified and the denture suitably modified to prevent any impingement. Further, since the denture prosthesis is being worn by the patient both during day and night, intaglio surface hygiene is of critical value and the patient must be suitably counselled on denture hygiene procedures. Increased vertical separation of the maxilla and the mandible when the dentures are used as MAD may cause initial discomfort, which is easily overcome by the patient in a couple of days.

The reason for setting the age limit of $61 \pm 4$ years for the study population was the higher chance of central sleep apnea in patients over this age, which could have otherwise provided false results. Gender distribution in the study was $80 \%$ men and $20 \%$ women. OSA is 3 to 4 times more prevalent in men than women, which may be related to differences in sex hormones and preferential fat distribution in women in the subcutaneous tissue of hips and thighs, with less deposition in the neck, in contrast to men. . $^{17,18}$

The efficacy of MAD in the treatment of edentulous patients with OSA has been reported in previous studies. In a study by Arisaka et al. comprising 34 OSA patients $(\mathrm{AHI} \geq 5$ ), the mean AHI in patients sleeping with dentures was lower than that of those without dentures $(13.3 \pm 10.0$ versus $17.7 \pm 14.6$, $\mathrm{p}=0.022) .{ }^{13}$ The result showed that $70 \%$ of the individuals showed marked improvement in AHI after wearing dentures.
However, this study did not show the effect of denture advancement on sleep variables.

Erovigni et al. showed by cephalometric evaluation that wearing denture induces modifications in the position of the tongue, of the jaw, and of the pharyngeal airway space that can favor the reduction of apnea episodes. ${ }^{19}$

Recently, a few studies have shown that oral appliances could be used in partially and totally edentulous patients with OSA. ${ }^{20}$ Giannasi et al. applied an adjustable MAD on a maxillary edentulous patient with a dentate mandible. ${ }^{21}$ They reported a sharp decrease in AHI from 18.0 to 2.2/h. Nayar and $\mathrm{Knox}^{22}$ reported some advantages of an individually fabricated MAD on a totally edentulous patient with OSAS. They reported improvement of patient compliance based on subjective data (such as patient history) without polysomnography. Kurtulmus and Cotert fabricated a combination of TRD and MAD for a totally edentulous patient. ${ }^{23}$

\section{CONCLUSION}

The results indicate that within the limits of the present study, this specific MAD is effective in reducing sleep apnea by lowering the AHI and reducing snoring and oxygen desaturation events/h in a significant way along with improvement in sleep efficiency. The MAD has been well tolerated by all patients.

\section{REFERENCES}

1. Young TM, Dempsey J, Skatrud J, Weber S, Badr S. The occurrence of sleep-disordered breathing among middle-aged adults. New Engl J Med 1993;328:1230-5.

2. Garcia-Rio F, Racionero MA, Pino JM, et al. Sleep apnea and hypertension. Chest 2000;117:1417-25.

3. Sharma SK, Ahluwalia G. Epidemiology of adult obstructive sleep apnoea syndrome in India. Indian J Med Res 2010;131:171-5.

4. Lam JC, Sharma SK, Lam B. Obstructive sleep apnoea: definitions, epidemiology \& natural history. Indian J Med Res 2010;131:165-5.

5. Reddy EV, Kadhiravan T, Mishra HK, et al. Prevalence and risk factors of OSA in middle-aged urban Indians: a community based study. Sleep Med 2009;10:913-8.

6. Young T, Palta M, Dempsey J, et al. Burden of sleep apnea: rationale, design, and major findings of the Wisconsin Sleep Cohort Study. WMJ 2009;108:246-9.

7. Bucca C, Cicolin A, Brussino L, et al. Tooth loss and obstructive sleep apnea. Respir Res 2006;7:8.

8. Ancoli-Israel S, Gehrman P, Kripke DF, et al. Long term follow up of sleep disordered breathing in older adults. Sleep Med 2001;2:511-6.

9. Ancoli-Israel S, Kripke DF, Klauber MR, et al. Sleep disordered breathing in community dwelling elderly. Sleep 1991;14:486-95. 
10. Cistulli PA, Gotsopoulos H, Marklund M, Lowe AA. Treatment of snoring and obstructive sleep apnea with mandibular repositioning appliances. Sleep Med Rev 2004;8:443-57.

11. Chan AS, Cistulli PA. Oral appliance treatment of obstructive sleep apnea: an update. Curr Opin Pulm Med 2009;15:591-6.

12. Vanderveken OM, Devolder A, Marklund M, et al. Comparison of a custom-made and a thermoplastic oral appliance for the treatment of mild sleep apnea. Am J Respir Crit Care Med 2008;178:197-202.

13. Arisaka H, Sakuraba S, Tamaki K, Watanabe T, Takeda J, Yoshida $\mathrm{K}$. Effects of wearing complete dentures during sleep on the apneahypopnea index. Int J Prosthodont 2009;22:173-7.

14. Meyer JB, Knudson RC. Fabrication of prosthesis to prevent sleep apnea in edentulous patients. J Prosthet Dent 1990;63:448-51.

15. Almeida FR, Furuyama RJ, Chaccur DC, et al. Complete denture wear during sleep in elderly sleep apnea patients: a preliminary study. Sleep Breath 2012;16:855-63.

16. Keyf F, Ciftci B, Guven FS. Management of obstructive sleep apnea in an edentulous lower jaw patient with a mandibular advancement device. Case Reports in Dentistry 2014;2014:436904.

17. Kapsimalis F, Kryger MH. Gender and obstructive sleep apnea syndrome, part 2: mechanism. Sleep 2002; 25:412-9.

18. Jamieson A, Guilleminault C, Partinen M, Quera-Salva MA. Obstructive sleep apneic patients have craniomandibular abnormalities. Sleep 1986;9:469-77.

19. Erovigni F, Graziano A, Ceruti P, Gassino G, De Lillo A, Carossa S. Cephalometric evaluation of the upper airway in patients with complete dentures. Minerva Stomatol 2005;54:293-301.

20. Taner T, Saglam Aydintay B, Turkyilmaz I, Demir AU. The use of modified mandibular advancement appliance in the treatment of a partially edentulous patient with obstructive sleep apnea. Hacettepe Dishek Fak Derg 2007;31:82-7.
21. Giannasi LC, Magini M, Oliveira CS, Oliveira LV. Treatment of obstructive sleep apnea using an adjustable mandibular repositioning appliance fitted to a total prosthesis in a maxillary edentulous patient. Sleep Breath 2008;12:91-5.

22. Nayar S, Knox J. Management of obstructive sleep apnea in an edentulous patient with a mandibular advancement splint: a clinical report. J Prosthet Dent 2005;94:108-11.

23. Kurtulmus H, Cotert HS. Management of obstructive sleep apnea in an edentulous patient with a combination of mandibular advancement splint and tongue-retaining device: a clinical report. Sleep Breath 2009;13:97-102.

\section{SUBMISSION \& CORRESPONDENCE INFORMATION}

Submitted for publication March, 2014

Submitted in final revised form August, 2014

Accepted for publication August, 2014

Address correspondence to: Dr. Arvind Tripathi; MD 10, Sector C, Aliganj, Lucknow- 226024, Uttar Pradesh, India; E-mail: atrip2006@gmail.com

\section{DISCLOSURE STATEMENT}

This was not an industry supported study. The authors have indicated no financial conflicts of interest. The work was performed at the Department of Prosthodontics, Saraswati Dental College \& Hospital, Lucknow and Department of Pulmonary Medicine, King George's Medical University, Lucknow, India. There was no off-label or investigational use 



\title{
Oral Appliance Therapy and Atrial Fibrillation
}

\author{
Kathleen Bennett, DDS, Diplomate, ABDSM
}

Department of Internal Medicine Division of Pulmonary Sleep Medicine UC Health West Chester Hospital, Cincinnati, OH

\begin{abstract}
Atrial fibrillation (AF) is the most common sustained arrhythmia affecting more than two million Americans. Patients have shown significant improvement both pre and post ablation with the use of both medication and CPAP. Non-compliant or non-tolerant CPAP patients may benefit from oral appliance therapy. Case is presented of a patient with AF, who was treated with an oral appliance.
\end{abstract}

KEYWORDS: oral appliance

Citation: Bennett K. Oral appliance therapy and atrial fibrillation. Journal of Dental Sleep Medicine 2014;1(3):121-122.

A trial fibrillation (AF) is the most common sustained arrhythmia, affecting more than two million Americans. The prevalence of obstructive sleep apnea (OSA) in patients with atrial fibrillation is $32 \%$ to $49 \%$. AF prevalence is directly correlated with the severity of obstructive sleep apnea (OSA).,2 Mechanisms thought to be at least partly responsible for the interaction of AF and OSA include elevated intrathoracic pressure, increased autonomic nervous system activity, and inflammation. ${ }^{3}$ A trend towards increased AF recurrence has been described in patients with untreated OSA after treatment for $\mathrm{AF}^{4}$ The risk of AF recurrence is related to the duration of oxygen saturation $<90 \%$ and the magnitude of the desaturation. $^{3}$

Patients have shown significant improvement both pre and post-ablation with the usage of both medication and CPAP. ${ }^{1}$ The most important benefit of CPAP may be the reduction in the frequency of the large negative swings in intra thoracic pressure.

Support for the use of oral appliance therapy in OSA has risen with the recent publication research confirming the positive effect of oral appliance therapy on blood pressure and cardiovascular mortality.,6 Non-compliant or non-tolerant CPAP patients may benefit from oral appliance therapy.

As a dentist when assessing the position of the mandible during the titration, one of our tools is pulse oximetry. What information can be gained by frequent use of pulse oximetry?

What is most significant when assessing the pulse oximetry? Does the oxygen nadir, the oxygen desaturation index, or the duration of time spent below $90 \%$ saturation contribute to the atrial fibrillation? What is the best way to manage these patients?

A 66-year-old, overweight (BMI 36.1) male presented with moderate OSA (AHI 17). His medical history included insulin dependent diabetes, hypertension, hypercholesterolemia, and atrial fibrillation. He had cardiac ablation in December 2012. He reported no occurrences of flutter and that he "feels better" since the ablation. He was not able to tolerate CPAP. He was fitted with an oral appliance in April 2013 at an initial advancement of $6 \mathrm{~mm}$ of a total protrusive range of $10 \mathrm{~mm}$. Oximetry at the initial setting indicated suboptimal results, and the appliance was advanced to $8 \mathrm{~mm}$ and oximetry repeated. Pre- and post-treatment outcomes are shown in Table 1. A second posttreatment oximetry was done 7 months later with no further advancement.

The patient's cardiologist reviewed the pulse oximetry results, concluding that the reduction in the total number of desaturations was helpful and the hypoventilation related to the obesity was not significant enough to use nocturnal oxygen at this time. As of September 2014, no recurrences of AF have been reported. A follow-up PSG with the appliance in place has been ordered.

Management of these patients might include a log of the time and circumstances of arrhythmic episodes. Aggressive advancement of the mandible to $60 \%$ to $70 \%$ of protrusion, and close communication with the patient at biweekly intervals may allow for more rapid reduction in symptoms. Regular nocturnal monitoring with pulse oximetry at different points of titration, and frequent communication with the cardiologist helps to improve outcomes. Patients are very grateful when they can finally sleep without the disruption of the atrial fibrillation episode.

Follow-up overnight PSG with the appliance in place after the airway is maximized is critical to determine the effectiveness.

\section{REFERENCES}

1. Loomba RS, Arora R. Obstructive sleep apnea and atrial fibrillation: a call for increased awareness and effective management. Am $J$ Ther 2012;19:e21-4.

Table 1-Pre and post-treatment outcomes.

$\begin{array}{lc} & \text { Baseline } \\ \text { AHI/ODI } & 17 \\ \mathrm{O}_{2} \text { Nadir } & 76 \% \\ \% \text { time }<90 \% \mathrm{O}_{2} & 14.4 \% \\ \text { Mean } \mathrm{O}_{2} \text { Saturation } & \text { Not available }\end{array}$

Oximetry at $\mathbf{6} \mathbf{~ m m}$
Advancement
14
$78 \%$
$40.2 \%$
$91 \%$

\begin{tabular}{cc} 
Oximetry at $\mathbf{8}$ mm & Advancement \\
\hline Initial & $\mathbf{7}$ months later \\
10 & 5 \\
$80 \%$ & $81 \%$ \\
$51.8 \%$ & $16 \%$ \\
$91 \%$ & $92 \%$
\end{tabular}


2. Digby GC, Baranchuk A. Sleep apnea and atrial fibrillation; 2012 update. Curr Cardiol Rev 2012;8:265-72.

3. Vanderveken OM, Boudewyns A, Ni Q, et al. Cardiovascular implications in the treatment of obstructive sleep apnea. J Cardiovasc Transl Res 2011;4:53-60.

4. Ng CY, Liu T, Shehata M, Stevens S, Chugh SS, Wang X. Meta-analysis of obstructive sleep apnea as predictor of atrial fibrillation recurrence after catheter ablation. Am J Cardiol 2011;108:47-51.

5. Phillips CL, Grunstein RR, Darendeliler MA, et al. Health outcomes of continuous positive airway pressure versus oral appliance treatment for obstructive sleep apnea: a randomized controlled trial. Am J Respir Crit Care Med 2013;187:879-87.

6. Anandam A, Patil M, Akinnusi M, Jaoude P, El-Solh AA. Cardiovascular mortality in obstructive sleep apnoea treated with continuous positive airway pressure or oral appliance: an observational study. Respirology 2013;18:1184-90.

\section{SUBMISSION \& CORRESPONDENCE INFORMATION}

Submitted for publication September, 2014

Accepted for publication September, 2014

Address correspondence to: Kathleen M. Bennett, DDS, UC Health Medical Arts Bldg., 222 Piedmont Ave., Suite 8300, Cincinnati OH 45219; Tel: (513)

871-9111; Fax: (513) 467-0943; E-mail: kmbennettdds@gmail.com

\section{DISCLOSURE STATEMENT}

Dr. Bennett has indicated no financial conflicts of interest. 


\title{
Current Barriers and Study Needs for Oral Appliance Therapy: The Personal Perspective of a Physician and Dentist
}

\author{
Bernard Fleury, MD; Alan A. Lowe, DMD, PhD²; ORal Appliance Network for Global Effectiveness Group \\ ${ }^{1}$ Centre d'Evaluations et de Traitements des Troubles du Sommeil, Hôpital Saint Antoine-Hôpitaux Universitaires Est Parisien-Université, Paris, \\ France; 'Professeur invité, UFR d'Odontologie University Paris Diderot, Division of Orthodontics, Faculty of Dentistry, University of British Columbia
}

\begin{abstract}
From the perspectives of a physician and a dentist, this review article summarizes the major barriers which appear to restrict the increased use of oral appliances in comparison to CPAP for the treatment of sleep disordered breathing. Barriers related to organizational issues, industrial development, differing effectiveness, contraindications, and side effects are discussed in detail. In addition, the perceived barriers between the dental and medical professions at large are outlined. Patients should actively participate in the choice of treatment after they have been informed of the benefits and limitations of both therapies.
\end{abstract}

KEYwORDs: oral appliance, obstructive sleep apnea, CPAP

CitAtion: Fleury B, Lowe AA, ORal Appliance Network for Global Effectiveness Group. Current barriers and study needs for oral appliance therapy: the personal perspective of a physician and dentist. Journal of Dental Sleep Medicine 2014;1(3):123-127.

$\mathrm{D}$ ue to its high prevalence and multiple consequences, the obstructive sleep apnea syndrome (OSAS) is a major public health problem. In a recently published study, this problem in France was addressed among 12,203 adults representative of the general population. ${ }^{1}$ The prevalence of symptoms highly suggestive of OSAS such as snoring most nights plus witnessed apneas and/or excessive daytime sleepiness (Epworth Sleepiness Scale score $>10$ ) was estimated at $4.9 \%$, which is close to the prevalence reported in the epidemiological literature. ${ }^{2}$ This prevalence reached $8 \%$ in hypertensive patients and $11 \%$ in obese subjects; only $15 \%$ of these subjects with a high probability of having OSAS who require treatment in our sample had undergone a sleep study. This underdiagnosis and undertreatment of OSAS is currently reported across the international medical literature. ${ }^{3}$

However, in 1981 effective treatment for sleep apnea was first identified when Collin Sullivan proposed a simple device called continuous positive airway pressure (CPAP) to alleviate the pharyngeal occlusion which occurred during sleep. ${ }^{4}$ Room air applied through the nares with a continuous positive pressure provided a pneumatic splint for the nasopharyngeal airway and was a safe and incredibly clever treatment for the OSAS syndrome. But, concurrently with the Sullivan publication, two studies reported the use of oral appliances (OA) to maintain the stability of the upper airway in OSAS patients. Cartwright and Samelson published the first description of the beneficial action of a tongue retaining device in $1982,{ }^{5}$ and three years later, Soll and George published a brief note on the effectiveness of a modified one piece mandibular advancement appliance in a series of five patients. ${ }^{6}$ Since then, hundreds of reports have been published in the literature about similar OAs. Nevertheless, some thirty years later, CPAP is accepted as the standard treatment for OSAS and OAs are often used primarily as an alternate therapy when CPAP fails or is not tolerated by the patient. For instance, some 400,000 OSAS patients in France are chronically treated with CPAP and 75,000 new CPAP patients are fitted every year. However, only 15,000 patients are treated long term with OAs, with approximately 3,500 new OA patients added every year.
Why does such a huge difference exist between CPAP and OA use after all these years and the incredible amount of published data that support OA efficacy? What are the possible barriers to the prescription of an OA compared to the prescription of a CPAP machine?

We have identified six major barriers to the development of OA therapy:

1. Barriers related to organizational issues

2. Barriers linked to industrial development

3. Barriers linked to different effectiveness

4. Barriers related to contraindications

5. Barriers related to side effects

6. Barriers between dental and medical professionals

\section{Barriers related to organizational issues}

For more than two decades, the effects of CPAP on sleep disordered breathing in all stages of sleep, including REM sleep, and in the supine body position was determined by a sleep technician during full-night polysomnography in a sleep laboratory. Today, it is very easy to prescribe a CPAP machine. The use of auto-titrating positive pressure (APAP), with comparable efficiency to that of fixed pressure machines, allows one to simplify the care and avoid a night in the sleep lab. ${ }^{7}$ If the practitioner wishes, one can choose a fixed pressure from APAP records obtained over a few weeks of treatment. According to the local insurance reimbursement guidelines, the patient either buys the device and mask directly or they are provided by the social security system via a health homecare provider, as in France for instance. Finally, for the sleep physician, a prescription or a phone call is sufficient to start the treatment. An ambulatory follow-up, after 1 or 2 months of treatment and every year thereafter, is often sufficient to provide adequate care.

To prescribe an $\mathrm{OA}$ is much more complicated. After the sleep recording, the sleep physician must refer the patient to a dentist with training in dental sleep medicine who will verify the absence of any intraoral contraindications, select one appliance suitable for the specific patient, and obtain impressions 
of the dentition and a bite registration to be sent to a laboratory. After one to three weeks, the definitive OA is obtained and the dentist will adjust the appliance in the patient's mouth to make it comfortable to wear. The titration procedure of slowly advancing the mandible will then begin, the efficacy of which is usually monitored by a sleep recording. It can take several weeks or months and a number of appointments with the dentist. ${ }^{8}$ Finally, follow-up should take place once a year by the attending physician and twice a year by the dentist. It is easier and faster and less labor-intensive to prescribe a CPAP device than an OA.

\section{Barriers linked to industrial development}

From the outset, industry has been interested in the manufacture of CPAP. From 1985, the companies SEFAM and Pierre Medical were created in France; Respironics and DeVilbiss appeared in the USA; ResMed in Australia; and since then many others have started manufacturing around the world. Over the years, the engineers of Pierre Medical, ${ }^{9}$ ResMed, ${ }^{10}$ and other companies have developed algorithms allowing for auto-titration of effective pressure. Industrial grouping has created marketing giants on the five continents. For any CPAP device, the therapeutic principle to establish a positive pressure in the upper airways is the same. The equivalence of the different CPAP devices is complete for a fixed pressure. The algorithms piloting the APAP vary model by model, but globally their efficiency is satisfactory and a simple modification of the range of pressures chosen initially allows one to correct the SDB. Masks that were initially customized for each patient are today industrially designed and offer a large choice to facilitate implementation of the treatment.

In contrast, OA manufacturing is a more recent phenomenon, which has no scale comparable to the industrial process with CPAP. During many years, more than 100 OAs have been described by a number of inventors. Many were derived from traditional orthodontic appliances, and their design, volume, and structural components differ. Even the principle of action on the mandible, tongue, or both differ. For many appliances, the manufacturing process has not been standardized. ${ }^{11}$ More recently, validated OA manufacturing has emerged according to laboratory controlled processes both in North America and internationally.

\section{Barriers linked to different effectiveness}

Today the effectiveness of a treatment is judged according to the principles of evidence-based medicine. This requires evaluation protocols that are rigorous, prospective, and randomized. To discuss the individual effectiveness of CPAP and OA and to compare their effectiveness, we rely on the systematic analysis of the literature carried out by the Cochrane group. A systematic review has been devoted to each treatment. In 2006, 36 trials were selected to evaluate CPAP, including 1,718 subjects. ${ }^{12}$ In 2006, only 17 trials were included to evaluate OAs (including 846 subjects), that were considered methodologically satisfactory by the very demanding group of experts of the Cochrane group. ${ }^{13}$ What were the results of their analyses as to the effectiveness of CPAP or OA on the objective parameters of a polysomnographic recording? When the efficacy of CPAP and $\mathrm{OA}$ is evaluated on the same patients, the reduction in AHI is systematically greater with CPAP. Whether from crossover or parallel arm studies, the apnea-hypopnea index (AHI) appears to be lower in the group treated with effective CPAP. However, the Cochrane data did not differentiate between single jaw position and titratable OAs, which has a major impact on efficacy. When a titrated OA is compared to a simple splint, the AHI is always lower in the titrated group. For AHI, when mandibular advancement devices were compared with devices that did not advance the mandible, there was a significant effect in favor of active advancement when compared to controls.

This overall superiority in effectiveness of CPAP over OA encompasses a more complex reality. Although CPAP is mechanically effective, three groups of responders to OA can be distinguished as reported in Chest in 2004. ${ }^{14}$ After careful OA titration, more than half of patients will have their AHI brought to a level comparable to that obtained with CPAP, while about one-quarter will be treatment failures. Between these two groups, one-quarter of patients decreased their AHI by over $50 \%$. This difference in effectiveness is easy to understand if we analyze the mode of action of the two treatments. CPAP increases the overall volume of the upper airway. The level of pressure required to open the airway depends on the subject, but is usually within a reasonable range of pressures that are tolerable to the patient. There is limited mechanical failure with CPAP.

The mode of action of an OA is more complex. Chan et al. reported that mandibular advancement increases the total airway volume predominantly by increasing the volume of the velopharynx..$^{15}$ More recently, Brown and al. described a complex movement of the airway associated with a lateral movement of the lateral wall of the nasopharynx via its connections to the mandible. ${ }^{16}$ This was associated with an anteroposterior elongation of the tongue overall and a rostrocaudal compression of the posterior tongue. On sequential MRI sections performed during progressive mandibular advancements, one can see the hyoid bone moves upward, resulting in a flattening of the floor of the mouth and a widening of all segments of the pharynx. This upward movement of the hyoid bone was described by Battagel et al., who emphasized its wide variation both in the amount and direction of movement between subjects. ${ }^{17}$ Depending on the subject's anatomy, the level of advancement leading to widening of the pharynx may be different. Movement of tissues with OAs is influenced by both anatomical and physiological differences between patients.

OSAS is a chronic disease whose effects on alertness or mood decrease the quality of life of patients. ${ }^{18}$ The suppression of abnormal respiratory events by OA or CPAP and their consequences on sleep architecture improves the quality of life of patients. ${ }^{12,13}$ Both therapies are only palliative. Which procedure finally brings the greatest improvement in the quality of life? Recently, the Cistulli group compared health effects after one month of optimal CPAP and OA therapy in OSA subjects with a randomized crossover design. ${ }^{19}$ Cardiovascular (24-h blood pressure, arterial stiffness), neurobehavioral (subjective sleepiness, driving simulator performance), and quality of life (FOSQ, SF-36) indices were compared between treatments in 126 patients with moderate-to-severe OSAS. Important health outcomes were similar after one month of optimal OA and CPAP treatment. Less discomfort associated with an $\mathrm{OA}$ and the close effectiveness on alertness probably explain the results of several studies, which most often conclude a similar improvement in quality of life with OA and CPAP. It could explain why, with equivalent effectiveness, the vast majority of patients prefer an $\mathrm{OA} .^{20}$ 
Adherence with treatment is a key factor, because a less effective OA worn for longer time periods could ultimately have an identical efficacy on the consequences of OSAS. Adherence with OA is likely to be better than adherence with CPAP. Vanderveken et al. recorded an overall objective mean adherence of OA use to be 6.6 hours per night at the 3-month followup, and they calculate the mean disease alleviation (MDA) as the product of objective compliance and therapeutic efficacy. ${ }^{21}$ MDA serves as a measure of the overall therapeutic effectiveness and turned out to be $51.1 \%$ with $\mathrm{OA}$, a value comparable to the MDA reported with CPAP. ${ }^{22}$ In the near future, RCT studies will integrate $\mathrm{OA}$ adherence monitors to answer these crucial issues over longer time periods.

\section{Barriers related to contraindications}

Contraindications for OAs are determined in part by the specific appliance used and the patient's overall dentition and dental health. OAs can only be used in patients able and willing to use an OA and are occasionally not tolerated because of claustrophobia issues, although clinicians who have experience with both therapies report much less so than with CPAP. Current practice parameters suggest that OAs are indicated in patients with primary snoring and mild to moderate OSA. ${ }^{23}$ They are not generally used in patients with central apnea. OAs have been contraindicated in severe OSA patients unless an unsuccessful trial of CPAP has been completed, but more recent data challenge this specific guideline. ${ }^{19}$ OAs should be selected, fitted, and adjusted by qualified dentists who are adequately trained in the field and not by individuals who are not licensed by their local regulatory authority to do so. Mild to moderate TMJ dysfunction is not currently recognized as a contraindication to OA therapy. OAs are commonly used in patients with at least eight teeth in each arch and no limitation in vertical and/or AP jaw position. The ability to comfortably reposition the jaw forward is absolutely mandatory, as is the requirement to adequately open the mouth vertically to allow for placement of the OA. ${ }^{24,25}$

In subjects who are edentulous, do not have at least eight teeth in both arches, have severe limitations in the anteroposterior position of the mandible, or who have significant periodontal disease, traditional OAs may be contraindicated. Instead an appliance such as a tongue repositioner/stabilizer ${ }^{26}$ could be used, which delivers less force directly to the teeth and distributes the pressure over other intraoral sites. However, tongue retaining devices are generally contraindicated in obligate mouth breathers, since it may be difficult to breathe through the mouth with these appliances.

\section{Barriers related to side effects}

Short-term side effects, similar to the insertion of any new dental appliance/prosthesis placed in the mouth, may include excessive salivation, dry mouth (especially in obligate pretreatment nasal breathers), and tooth discomfort. Minor soft tissue discomfort can also occur during the first few weeks of OA wear. Lack of adequate retention is primarily an appliance design issue, dependent in part on which of the OAs currently available on the market is being used and how it has been manufactured. Either too little or too much retention may be a contraindication for a specific appliance. Initial transient side effects usually resolve within several days to several weeks with minor adjustments by the dentist and continued adaptation to the OA by the patient.

More significant side effects may occur during the OA titration stage, which may in some patients continue over several months as the mandible is gradually moved forward. These may include minor tooth movement, occlusal contact changes, soft tissue issues, and TMJ/myofascial discomfort. A sense of the teeth not touching in the morning is reported in some patients. Initial jaw discomfort is often an indication of repositioning the mandible too far forward. Initial titration side effects, if not readily recognized and managed by the dentist, can be a significant deterrent for continued use of an OA in some patients and is one of the main reasons for the need for frequent appointments during this stage of therapy.

Long-term OA side effects (potential tooth movement and myofascial issues) have been well documented. ${ }^{26-28}$ Some $14 \%$ of OSA patients who had worn a mandibular repositioning appliance for five years or longer were found to have no change in their occlusion, $42 \%$ had a favorable change, and $44 \%$ had an unfavorable change. ${ }^{27}$ Patients with larger overbites and overjets had the most favorable occlusal changes based on cephalometric and study model analyses. Mandibular arch width increased more than maxillary arch width, crowding decreased in both arches, the curve of Spee became flat in the premolar area, the mandibular canine to second molar segment moved forward in relation to the maxillary arch, the bite opened and the overjet decreased. Both jaw exercises and morning repositioning splints have been suggested as potential therapies to reduce occlusal changes when they occur. The most common tongue appliance-induced dental changes included anterior and/or unilateral posterior open bites and reduced anterior overjets. ${ }^{26}$ Two possible mechanisms for the TRD side effects have been suggested-one is the forward pressure of the tongue on the anterior dentition and the other is the lateral pressure of the tongue directly on the posterior arch.

Even unfavorable dental side effects should not be considered a contraindication to OA therapy, since occlusal changes are usually readily adapted to by patients when compared to the consequences of life-threatening OSA. It is also important to recognize that two years of nasal CPAP wear may also cause dental and skeletal changes due to the pressures exerted by the mask and straps on the anterior maxilla. ${ }^{29}$ After nCPAP use, cephalometric variables demonstrated a significant retrusion of the anterior maxilla, a decrease in maxillary-mandibular discrepancy, a setback of the supramentale and chin positions, a retroclination of maxillary incisors, and a decrease in facial convexity. In summary, both OAs and CPAP have definable long-term effects on dentition.

There appears to be no evidence of consistent undesirable long-term effects of OAs on the TMJ. Indeed, it appears that the intensity of TMJ symptoms decreases significantly throughout treatment in patients who use their OA regularly due possibly to the unloading of the joint when the condyle is held down and out of the fossa by the OA and/or the resolution of tooth grinding observed subsequent to improved sleep parameters. A crossover study of an adjustable OA and a mandibular occlusal splint in sleep bruxism patients found that an OA reduced sleep bruxism episodes to a greater extent than a splint alone. ${ }^{30}$ If myofascial problems due to the jaw not settling back to its regular position 
throughout the day are not resolved during the first few weeks, specific jaw exercises (jig exercise and stretching movements) may reduce the occlusal functional impairment. ${ }^{31}$ Both exercises produced significant increases in occlusal contact area and bite force in the morning compared with periods of no exercise. There was no significant difference between the two exercises, although the jig exercise tended to be more effective in the anterior region while the stretching movements tended to more effective in the molar region. Using MRI, OAs did not result in any observable remodeling of the TMJ. Remodeling was not observed after 11.5 months, which suggests that the appliance does not significantly alter the TMJ, but long-term studies with a greater sample size are required to determine whether there is a remodeling of the TMJ or neighboring structures after two or more years. ${ }^{32}$

Different side effects have been documented for thermoplastic boil and bite type OAs (especially those only available in one preformed size) when compared to custom-made titratable appliances. Difficulty in optimum fit has been documented, and "uncomfortable" appears to be the most common reason to stop wearing a boil and bite appliance. ${ }^{33}$ A clinical trial where both types of appliances were used in the same patients for a period of four months identified that the AHI was only reduced with the custom-made device and that it also reduced snoring to a greater extent than the thermoplastic device. ${ }^{34}$ The success rate was higher with the custom-made device (60\% vs. 31\%). One-third of the patients demonstrated adherence failure with the thermoplastic device mainly because of insufficient overnight retention. Total failure rate with the thermoplastic device was $69 \%$; the majority (63\%) of these were successfully treated with the custom-made device. At the end of the study, a large majority of the patients chose the custom-made device.

\section{Barriers between dental and medical professionals}

Based on our joint clinical experiences as a sleep physician and an orthodontist over the past three decades, it appears that a number of barriers between the dental and medical professions have slowed down the wide acceptance of OAs for the treatment of OSAS. Although both groups are credentialed by their respective professional organizations, dentists often receive their education in sleep from continuing dental education courses and oral appliance manufacturers whereas physicians can obtain formal training as sleep specialist and from those CPAP companies who also market oral appliances. Dentists often see patients of various ages in a clinical setting where they may suspect sleep disordered breathing. Sleep physicians traditionally operate referral practices. Dentists depend on a medical diagnosis from a physician and, on occasion, use ambulatory monitoring as an aid to titration. In contrast, physicians routinely utilize both polysomnographic studies and ambulatory monitors. Dentists often refer patients to physicians for sleep issues and physicians on occasion refer patients to dentists. Appliance choices are made by dentists based on continuing education courses and oral appliance manufacturers. Physicians are more likely to learn of oral appliance types from the literature or CPAP suppliers. As to titration tools, it may take several months for a dentist to titrate an oral appliance based on symptomatic improvement or ambulatory monitoring. Physicians often utilize polysomnography or autoPAP for CPAP titration. Payment for oral appliance follow up may be an insurance issue in many jurisdictions. Dentists readily observe and manage oral appliance side effects, but physicians may not understand the significance of occlusal changes and generally are unaware of the long-term effects of oral appliances on dentition. Outcome measurements differ between dentists, who use snoring and symptomatic improvement to reduce the AHI to less than $10 / \mathrm{h}$, and physicians who utilize both polysomnographic and ambulatory monitoring and often look for a reduction in AHI to less than $5 / \mathrm{h}$, similar to what they use with CPAP. Physicians also emphasize improved oxygenation and decreased arousals when they evaluate outcomes and generally have lower expectations of oral appliance effectiveness. Dentists generally follow their patients annually and replace the oral appliances every three to five years, whereas physicians traditionally respond to patient requests for recall appointments, and the CPAP provider is more actively involved in the long-term maintenance of the patient. From a patient management perspective, some or all of these factors have been shown to be barriers between the medical and dental professions when oral appliances are used.

\section{Future Considerations}

Based on this analysis of the barriers to the continued development of OA use, we would like to make several proposals for the future. OA therapy has a definite place in the treatment decision-making process for OSAS. A better knowledge by sleep physicians of the current data on the efficiency of OA therapy according to principles of evidence-based medicine is required. Tests of OA titration efficacy with a cost-effective portable monitoring system as used by some dentists could be an elegant way to convince the sleep physician to prescribe future OAs after being reassured of their efficacy. In addition, the sleep community has to admit that if CPAP remains the reference treatment of OSAS, it is only effective if it is worn during adequate time periods every night. Clinicians who routinely utilize both therapies report that long term adherence with CPAP is a major issue and that the adherence with OA appears to be superior. However, randomized controlled trials to compare objective adherence for both $\mathrm{OA}$ and CPAP patients are required to accurately quantify these differences. It could be debated whether greater adherence with a less effective treatment might lead to a similar result in term of vigilance or cardiovascular outcomes. The place of CPAP as reference treatment could be challenged. The patient, informed about the benefits and limitations of the two treatments, should participate in the choice of treatment, even in the case of severe OSA. Apart from those patients with a major impairment in alertness that puts them or the community at risk or patients with unstable cardiovascular disease, for the majority of cases we have the opportunity to choose the most appropriate treatment. ${ }^{35}$

In all cases, it is necessary to improve the options offered to the patient as to the possibility of being treated with an OA. In the future, it is therefore obligatory to include sleep medicine in the undergraduate education of both dentists and physicians, preferably in a collaborative way. Hand in hand with that development, the creation and enhancement of specific academic and nonacademic training programs in dental sleep medicine are highly desired in order to significantly increase the number of trained dentists able to adequately respond to the requests of sleep physicians. This will promote the communication between sleep physicians and dental sleep practitioners. 
Finally, these barriers for the prescription of OAs are likely to have different impacts according to specific countries or continents. If we wish to better establish OA therapy in the therapeutic strategy of OSAS, it is imperative that we understand the place of OA in the therapeutic strategies around the world. It is in this spirit that an international group of trained practitioners from the USA, Canada, Japan, and Europe met under the auspices of the AADSM to construct ORANGE36, a large cohort of OA patient data that will allow us to gradually answer the questions that we ask ourselves today.

\section{REFERENCES}

1. Furhman C, Fleury B, Nguyen XL, Delmas MC. Symptoms of sleep apnea syndrome: high prevalence and underdiagnosis in the French population. Sleep Med 2012;13:852-8.

2. Young T, Palta M, Dempsey J, Peppard PE, Nieto FJ, Hla KM. Burden of sleep apnea: rationale, design, and major findings of the Wisconsin Sleep Cohort study. WMJ 2009;108:246-9.

3. Mold JW, Quattlebaum C, Schinnerer E, Boeckman L, Orr W, Hollabaugh K. Identification by primary care clinicians of patients with obstructive sleep apnea: a practice-based research network (PBRN) study. J Am Board Fam Med 2011;24:138-45.

4. Sullivan CE, Issa FG, Berthon-Jones M, Eves L. Reversal of obstructive sleep apnoea by continuous positive airway pressure applied through the nares. Lancet 1981;18:862-5.

5. Cartwright RD, Samelson CF. The effects of a nonsurgical treatment for obstructive sleep apnea. The tongue-retaining device. JAMA 1982;248:705-9.

6. Soll BA, George PT. Treatment of obstructive sleep apnea with a nocturnal airway-patency appliance. N Engl J Med 1985;313:386-7.

7. Nolan GM, Doherty LS, Mc Nicholas WT. Auto-adjusting versus fixed positive pressure therapy in mild to moderate obstructive sleep apnoea. Sleep 2007;30:189-94.

8. Almeida FR, Lowe AA. Principles of oral appliance therapy for the management of snoring and sleep disordered breathing. Oral Maxillofac Surg Clin North Am 2009;21:413-20.

9. Meurice JC, Marc I, Sériès F. Efficacy of auto-CPAP in the treatment of obstructive sleep apnea/hypopnea syndrome. Am J Respir Crit Care Med 1996;153:794-8.

10. Gagnadoux F, Rakotonanahary D, Martins de Araujo MT, Barros-Vieira $S$, Fleury B. Long-term efficacy of fixed CPAP recommended by Autoset for OSAS. Sleep 1999;15:1095-9.

11. Ahrens A, McGrath C, Hägg U. A systematic review of the efficacy of oral appliance design in the management of obstructive sleep apnoea. Eur J Orthod 2011;33:318-24.

12. Giles TL, Lasserson TJ, Smith BH, White J, Wright J, Cates CJ. Continuous positive airways pressure for obstructive sleep apnoea in adults. Cochrane Database Syst Rev 2006 Jul 19:CD001106.

13. Lim J, Lasserson TJ, Fleetham J, Wright J. Oral appliances for obstructive sleep apnoea. Cochrane Database Syst Rev 2006 Jan 25:CD004435.

14. Fleury B, Rakotonanahary D, Petelle B, et al. Mandibular advancement titration for obstructive sleep apnea: optimization of the procedure by combining clinical and oximetric parameters. Chest 2004;125:1761-7.

15. Chan AS, Lee RW, Srinivasan VK, Darendeliler MA, Grunstein RR, Cistulli PA. Nasopharyngoscopic evaluation of oral appliance therapy for obstructive sleep apnoea. Eur Respir J 2010;35:836-42.

16. Brown EC, Cheng S, McKenzie DK, Butler JE, Gandevia SC, Bilston LE. Tongue and lateral upper airway movement with mandibular advancement. Sleep 2013;36:397-404.

17. Battagel JM, Johal A, L'Estrange PR, Croft CB, Kotecha B. Changes in airway and hyoid position in response to mandibular protrusion in subjects with obstructive sleep apnoea (OSA). Eur J Orthod 1999;21:363-76.

18. Karkoulias K, Lykouras D, Sampsonas F, et al. The impact of obstructive sleep apnea syndrome severity on physical performance and mental health. The use of SF-36 questionnaire in sleep apnea. Eur Rev Med Pharmacol Sci 2013;17:531-6.
19. Phillips CL, Grunstein RR, Darendeliler MA, et al. Health outcomes of continuous positive airway pressure versus oral appliance treatment for obstructive sleep apnea: a randomized controlled trial. Am J Respir Crit Care Med 2013;187:879-87.

20. Gagnadoux F, Fleury B, Vielle B, et al. Titrated mandibular advancement versus positive airway pressure for sleep apnoea. Eur Respir J 2009;34:914-20.

21. Vanderveken OM, Dieltjens M, Wouters K, De Backer WA, Van de Heyning PH, Braem MJ. Objective measurement of compliance during oral appliance therapy for sleep-disordered breathing. Thorax 2013;68:91-6.

22. Ravesloot MJ, de Vries N. Reliable calculation of the efficacy of nonsurgical and surgical treatment of obstructive sleep apnea revisited. Sleep 2011;34:105-10.

23. Kushida C, Morgenthaler T, Littner M, et al. Practice parameters for the treatment of snoring and OSA with oral appliances: an update for 2005. Sleep 2006;29:240-3.

24. Chen H, Lowe A. Updates in oral appliance therapy for snoring and OSA. Sleep Breath 2013;17:473-86.

25. Cistulli P, Ferguson K, Lowe A. Oral appliances for sleep disordered breathing. In: Kryger M, Roth T, Dement W, eds., Principles and practice of sleep medicine. Elsevier Saunders, Quintessence, 2011:1266-77.

26. Chen H, Lowe AA, Strauss AM, de Almeida FR, et al. Dental changes evaluated with a 3D computer-assisted model analysis after long-term tongue retaining device wear in OSA patients. Sleep Breath 2008;12:169-78.

27. Almedia F, Lowe A, Otsuka R, et al. Long-term sequelae of oral appliance therapy in OSA patients. Part 2. Study model analysis. Am J Orthod Dentofacial Orthop 2006;129:205-13.

28. Marklund M, Franklin K, Persson M. Orthodontic side effects of mandibular advancement devices during treatment of snoring and sleep apnoea. Eur J Orthod 2001;23:135-44.

29. Tsuda H, Almeida F, Tsuda T, Moritsuchi Y, Lowe A. Craniofacial changes after two years of nCPAP use in OSA patients. Chest 2010;138:870-4.

30. Landry-Schonbeck A, de Grandmont P, Rompre P, Lavigne G. Effect of an adjustable mandibular advancement appliance on sleep bruxism: a crossover sleep laboratory study. Int J Prosthodont 2009;22:251-9.

31. Ueda H, Almeida F, Chen H, Lowe A. The effect of two jaw exercises on occlusal function in OSA patients during oral appliance therapy-a randomized controlled trial. Am J Orthod Dentofacial Orthop 2009;135:430-1.

32. de Almeida FR, Bittencourrt LR, de Almeida CI, Tsuiki S, Lowe AA, Tufik S. Effects of mandibular posture on OSA severity and the temporomandibular joint in patients fitted with an oral appliance. Sleep 2002;25:507-13.

33. Tsuda H, Almeida F, Masumi S, Lowe A. Side effects of boil and bite type oral appliance therapy in sleep apnea patients. Sleep Breath 2010;14:227-32

34. Vanderveken O, Devolder A, Marklund M, et al. Comparison of a custom-made and a thermoplastic oral appliance for the treatment of mild sleep apnea. Am J Respir Crit Care Med 2008:178:197-202.

35. Krucien N, Gafni A, Fleury B, Pelletier-Fleury N. Patients' with obstructive sleep apnoea syndrome (OSAS) preferences and demand for treatment: a discrete choice experiment. Thorax 2013:68:487-8.

36. Almeida F, Vanderveken O, Cistulli P, et al. ORal Appliance Network on Global Effectiveness (ORANGE): start-up and design description. Journal of Dental Sleep Medicine 2014;1;17-20.

\section{SUBMISSION \& CORRESPONDENCE INFORMATION}

\author{
Submitted for publication January, 2014 \\ Submitted in final revised form May, 2014 \\ Accepted for publication May, 2014
}

Address correspondence to: Bernard Fleury, MD, Centre d'Evaluations et de Traitements des Troubles du Sommeil, Hôpital Saint Antoine, 184 rue du Faubourg Saint Antoine-75012 Paris, France; E-mail: Bernard.fleury@ sat.aphp.fr

\section{DISCLOSURE STATEMENT}

This was not an industry supported study. Dr. Fleury holds an oral appliance patent. Dr. Lowe holds an adjustable oral appliance patent. 



\title{
Looking Through the Glass Ceiling
}

\author{
Commentary on Fleury B, Lowe AA, ORal Appliance Network for Global Effectiveness Group. Current barriers \\ and study needs for oral appliance therapy: the personal perspective of a physician and dentist. Journal of Dental \\ Sleep Medicine 2014;1(3):123-127.
}

\author{
Samuel T. Kuna, MD, FAASM
}

Philadelphia VA Medical Center, Philadelphia, PA

$\mathrm{D}$ espite growing evidence-based medicine demonstrating the efficacy and effectiveness of oral mandibular advancement appliance $(\mathrm{OA})$ treatment of patients with obstructive sleep apnea (OSA), the OA is still generally considered to be a secondary, alternative treatment to positive airway pressure (PAP). Current practice parameter recommendations limit the use of OA to patients with milder OSA who prefer OAs to PAP, or who do not respond to PAP, are not appropriate candidates for PAP, or who fail treatment attempts with PAP or treatment with behavioral measures such as weight loss or sleep position change. ${ }^{1}$ In the review paper "Current Barriers and Study Needs for Oral Appliance Therapy: The Personal Perspective of a Physician and Dentist" ${ }^{\prime 2}$ in this issue of the Journal of Dental Sleep Medicine, Dr. Lowe and Dr. Fleury present their opinions as to why OA has not gained wider acceptance and present their personal perspective about what is needed to break through this "glass ceiling." I will summarize those barriers, based on my own personal perspective, to be delayed time to achieve efficacious treatment, inability to predict whether OA treatment will achieve adequate control of sleep disordered breathing in a particular patient, inability to objectively monitor OA adherence, and the close partnership required between dental and medical professionals.

Two barriers listed by Dr. Lowe and Dr. Fleury that I will not consider are OA contraindications and side effects. Both OA and PAP treatment are contraindicated in some patients and are associated with side effects. Both, however, have minimal risk, and I personally do not regard these as reasons why practitioners prefer PAP to OA treatment. The authors of the paper also feel that lack of standardization of OA manufacturing is another barrier. The plethora of commercially available OAs differ widely in design; however, there is little evidence to support that one custom made OA is more efficacious than another., ${ }^{3,4}$ More studies comparing different OAs are needed, but it seems that there may be a wide latitude in OA design.

As stated by Dr. Lowe and Dr. Fleury, it is easier, faster, and less labor intensive to prescribe a PAP device than an OA. PAP is an "off-the-shelf" treatment. Once the diagnosis of OSA is established patients can be quickly started on PAP treatment. The growing use of automatically adjusting PAP (APAP) instead of continuous positive airway pressure (CPAP) has further reduced the time from diagnosis to efficacious treatment by obviating the need for a PAP titration to establish a fixed pressure setting. In contrast, it can take weeks to months to adjust an OA before the patient's sleep disordered breathing is under adequate control. Sleep specialists faced with a symptomatic patient, particularly those at risk for traffic and industrial accidents, are therefore more likely to recommend PAP treatment.

Another major barrier cited by Dr. Lowe and Dr. Fleury is the inability to predict whether $\mathrm{OA}$ treatment will achieve adequate control of sleep disordered breathing in a particular patient. Sleep specialists are unlikely to recommend OA, patients are unlikely to select $\mathrm{OA}$, and, most of all, insurers are unlikely to provide OA coverage when they will only know if the treatment is efficacious after weeks to months of OA management. A recent development that promises to address this barrier is the use of a remotely controlled mandibular positioning device during polysomnography to predict if mandibular advancement will be efficacious prior to initiation of OA treatment. ${ }^{5}$

Another major barrier mentioned in the review paper $^{2}$ is the inability of practitioners to objectively monitor OA adherence. Objectively monitored PAP adherence is being used by insurance carriers to require that its beneficiaries achieve a certain level of PAP usage to qualify for coverage. Similar standards are increasingly being adopted by the transportation industry for continued employment of its workers. Given these policies, patient use of OA will need to be measured objectively in order to compete with PAP. Recently developed datachips that are implanted in the OA and record temperature will address this barrier. ${ }^{6,7}$ However, this emerging technology is still not being widely used clinically. Hopefully, future studies with objectively monitored OA adherence will allow us to answer whether the presumed greater adherence to OA compared to PAP treatment is indeed true and offsets the decreased OA efficacy, thereby resulting in clinical outcomes comparable to PAP treatment.

Though the OA temperature sensor is likely to become a part of routine OA treatment in the near future, we still do not have a way to assess OA efficacy other than by performing a sleep study. In contrast, PAP downloads provide an estimation of the amount of sleep disordered breathing on treatment that is reasonably accurate. ${ }^{8}$ In addition, PAP manufacturers and other enterprises are rapidly adapting telehealth technologies to provide practitioners and patients ready access to adherence and efficacy information.

While noted near the end of the review paper, perhaps the most important requirement for OA treatment is a close collaboration between the sleep specialist and the dentist. In the "Future Considerations" section the authors state, "Tests of OA titration efficacy with a cost effective portable monitoring system as used by some dentists could be an elegant way to convince the sleep physician to prescribe future OAs after being reassured of their efficacy." While the use of home sleep testing is an important component of OA management, the dental community will fail 
to build collaborative ties if it takes this business away from the sleep physician.

An important barrier to OA treatment that is not mentioned in the paper is cost of OA treatment. No matter how much we improve OA treatment, its cost needs to be competitive to PAP treatment. The review paper by Dr. Lowe and Dr. Fleury tells us why OA treatment has not achieved greater acceptance and provides ways in which those barriers may be removed. Whether OA will ever achieve a usage equivalent to PAP treatment is debatable, but given recent advances, we can be optimistic that $\mathrm{OA}$ will continue to gain wider acceptance as an important treatment option for many patients with OSA.

\section{CITATION}

Kuna ST. Looking through the glass ceiling. Journal of Dental Sleep Medicine 2014;1(3):129-130.

\section{REFERENCES}

1. Kushida CA, Morgenthaler TI, Littner MR, et al. Practice parameters for the treatment of snoring and obstructive sleep apnea with oral appliances: an update for 2005. Sleep 2006;29:240-3.

2. Fleury B, Lowe AA, ORal Appliance Network for Global Effectiveness Group. Current barriers and study needs for oral appliance therapy: the personal perspective of a physician and dentist. Journal of Dental Sleep Medicine 2014;1:123-7.

3. Ahrens A, McGrath C, Hagg U. A systematic review of the efficacy of oral appliance design in the management of obstructive sleep apnoea. Eur J Orthodont 2011;33:318-24.
4. Sutherland K, Vanderveken OM, Tsuda H, et al. Oral appliance treatment for obstructive sleep apnea: an update. J Clin Sleep Med 2014;10:215-27.

5. Remmers J, Charkhandeh S, Grosse J, et al. Remotely controlled mandibular protrusion during sleep predicts therapeutic success with oral appliances in patients with obstructive sleep apnea. Sleep 2013;36:1517-25

6. Dieltjens M, Braem MJ, Vroegop AV, et al. Objectively measured vs selfreported compliance during oral appliance therapy for sleep-disordered breathing. Chest 2013;144:1495-502.

7. Vanderveken OM, Dieltjens M, Wouters K, De Backer WA, Van de Heyning PH, Braem MJ. Objective measurement of compliance during oral appliance therapy for sleep-disordered breathing. Thorax 2013;68:91-6.

8. Berry RB, Kushida CA, Kryger MH, Soto-Calderon H, Staley B, Kuna ST. Respiratory event detection by a positive airway pressure device. Sleep 2012;35:361-7.

\section{SUBMISSION \& CORRESPONDENCE INFORMATION}

Submitted for publication August, 2014

Accepted for publication August, 2014

Address correspondence to: Samuel T. Kuna, M.D., Philadelphia VA Medical Center (111P), Sleep Medicine Section, 3900 Woodland Avenue, Philadelphia, PA 19104; Tel: (215) 823-4400; Fax: (215) 823-5194; E-mail: skuna@mail.med.upenn.edu

\section{DISCLOSURE STATEMENT}

Dr. Kuna has indicated no financial conflicts of interest. 


\section{Rapid Maxillary Expansion for Pediatric Sleep Disordered Breathing}

Rose D. Sheats, DMD, MPH

Adjunct Associate Professor, Oral Facial Pain Group, Dental Sleep Medicine Unit, University of North Carolina School of Dentistry, Chapel Hill, NC

$\mathrm{R}^{2, a}$ apid maxillary expansion (RME), also known as rapid palatal expansion, is gaining interest in the medical and dental community as a potential therapeutic modality to treat sleep disordered breathing in pediatric patients. RME is an orthodontic procedure indicated for children who demonstrate a transverse deficiency in the width of their maxilla, usually manifested by the presence of a posterior crossbite.

Increase in the width of the maxilla is accomplished by placement of an expansion screw in the palate that is secured to the dentition. Generally RME appliances are deferred until the maxillary permanent first molars have erupted. Two-band expanders are secured to permanent first molars; 4-band expanders also incorporate either second primary molars or first or second premolars (Figure 1). The goal is to increase maxillary width by skeletal expansion (orthopedic) and not by dental expansion (orthodontic), but in reality both skeletal and dental expansion occur.

Activation of the expansion screw separates the two halves of the maxilla at the midline suture but also impacts the other circumaxillary sutures. The zygomaticomaxillary, zygomaticotemporal, zygomaticofrontal, nasomaxillary, and nasofrontal sutures are also impacted by this procedure. ${ }^{1,2}$ Occasionally patients report feeling pressure at the nasofrontal suture or other circumaxillary sutures when the expander is activated.

Sufficient expansion occurs in days to a few weeks, and a telltale sign of skeletal expansion is the appearance of a transient maxillary midline diastema (Figure 2). Typical expansion rates vary from $0.25 \mathrm{~mm}$ to $0.5 \mathrm{~mm}$ per day until the desired expansion is achieved. The maxilla is intentionally over-expanded to accommodate anticipated dental and skeletal relapse. ${ }^{3}$

Bone remineralization of the expanded suture requires 3-6 months, during which the expander must remain in place. ${ }^{4}$ To manage relapse when the expander is removed, ${ }^{3,5}$ another form of retention is indicated as discussed below.

As early as 1965, Haas described the ability of RME to increase nasal cavity volume and to improve nasal respiration, ${ }^{6}$ and other studies followed that investigated the association between RME and nasal airway resistance with variable results. ${ }^{7-10}$ These reports preceded the widespread medical recognition of sleep disordered breathing (SDB), and thus the technique was not advanced at the time as a treatment option for SDB.

In 2004 Pirelli and coworkers described a case series of 31 children diagnosed with obstructive sleep apnea syndrome (OSAS) whose AHI normalized after RME and remained stable at 4 months. ${ }^{11}$ Villa evaluated 14 OSAS children who underwent RME and demonstrated significant improvement in sleep parameters and symptoms of SDB at 12 months and again in 36 months in a follow-up study of 10 of the original 14 children. ${ }^{12,13}$ Limitations of these studies included the small sample size and absence of a control group, but they nevertheless demonstrated the potential value of the procedure in the management of pediatric SDB.

To date, no randomized clinical trials have been conducted to assess more rigorously the effect of RME on pediatric sleep disordered breathing. Studies are lacking to identify the optimal age for RME and to determine the stability of improvement in respiratory parameters, the effect on behavioral and cognitive outcomes, and the long-term impact on health outcomes.

\section{Patient Selection}

The following criteria must be considered in determining the most appropriate patients for RME:

1. Maxillomandibular transverse relationships

2. Mid-palatal suture patency

3. Anatomy of dental crowns that will retain the fixed device

4. Ability of patient to tolerate dental procedures and impressions as well as a bulky intra-oral appliance fixed to the maxillary dentition

\section{Maxillomandibular Transverse Relationships}

Orthodontic expansion of the maxilla is undertaken in the presence of maxillary constriction. This is generally revealed by either a unilateral or bilateral posterior crossbite. Often unilateral crossbites represent a symmetric bilateral maxillary constriction in which the patient compensates by shifting the mandible to one side or the other to establish occlusal contacts ("functional shift") (Figure 3). This can be readily seen by the presence of non-coincident midlines but must be confirmed by ascertaining the mandibular shift to distinguish from a true unilateral maxillary constriction.

In some instances the mandibular dentition masks a maxillary constriction by lingual tipping of the mandibular posterior teeth to compensate. The classic crossbite will not be present, and a decision must be made regarding the feasibility of correcting the mandibular constriction as well. Efforts to expand the mandible are limited to what can be accomplished by dental expansion as skeletal expansion of the mandible is more challenging and has met with more limited success.

\section{Mid-Palatal Suture Patency}

Palatal expansion is most effectively accomplished in patients whose mid-palatal suture is still patent. This suture fuses, or is resistant to expansion, around puberty ${ }^{14}$; thus prepubertal and adolescent patients are most desirable. Adults can also be expanded by RME; however, the procedure requires surgical release of not only the palatal suture but also the maxillary circumferential sutures and the pterygoid plates, a technique known as surgically assisted rapid palatal expansion. ${ }^{15}$ It approximates a Le Fort I osteotomy procedure without the down-fracture of the maxilla and is therefore not a trivial procedure for an adult. 

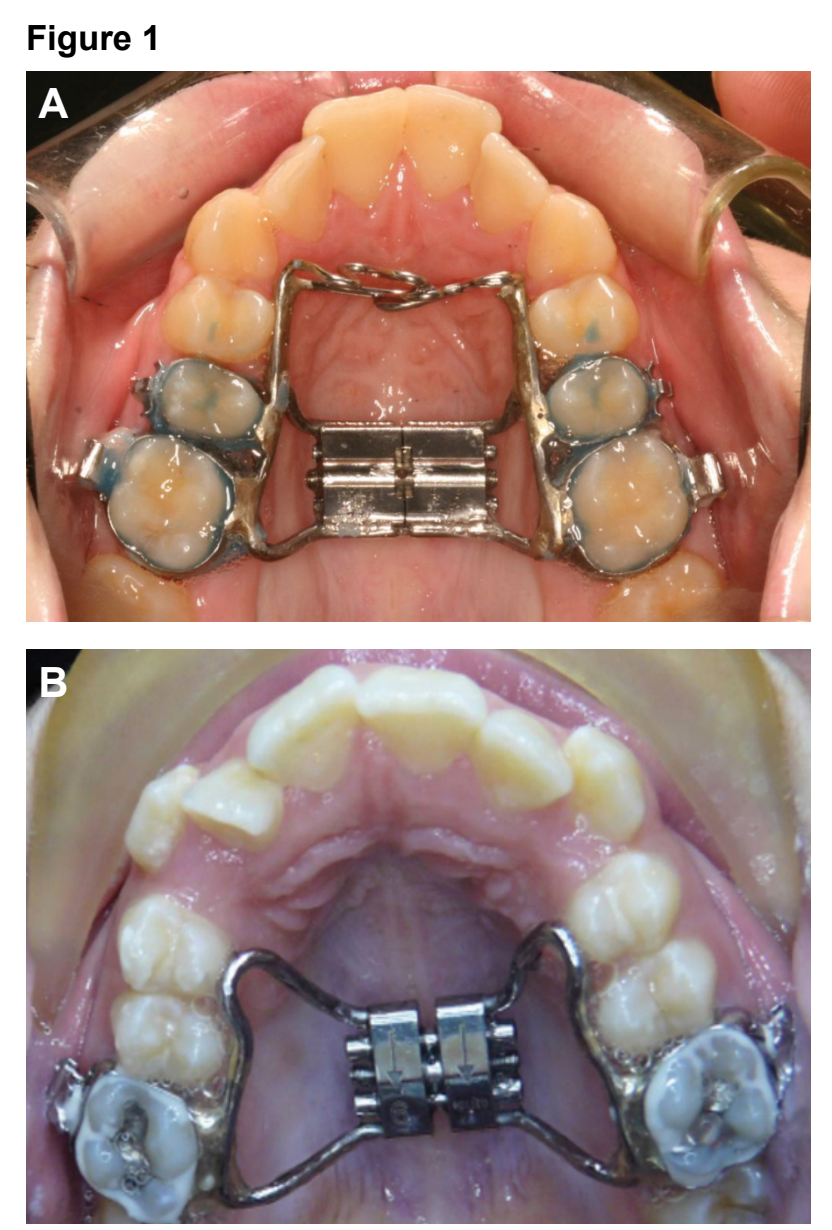

(A) 4-band rapid maxillary expander. (B) 2-band rapid maxillary expander. Image courtesy of John Mark Griffies, DDS

\section{Crown Anatomy}

Crown anatomy is critical to minimize dislodging of the appliance during treatment. The decreased crown height and conical shape of the primary dentition do not favor adequate band adaptation and retention. Furthermore, exfoliation of deciduous teeth during the expansion or the retention phases could jeopardize the procedure.

If crown anatomy is not conducive to retaining bands, an alternative design of the expander includes acrylic occlusal coverage of the dentition. ${ }^{5}$ The expander is cemented onto the occlusal and/or buccal surfaces of the teeth which necessitates practitioner vigilance to monitor for cement leaks that may lead to demineralization and caries formation under the appliance. Such appliances, if secured to deciduous teeth, may also impede exfoliation of the primary dentition and delay emergence of the permanent successor. This concern is generally of minor consequence, however, as the permanent tooth will erupt shortly after the appliance is removed. Patients and parents must be alerted to the possibility that deciduous teeth may dislodge upon removal of such an expander.

\section{Tolerance of Technical Procedures and Intra-Oral Appliance}

The age at which a young patient may be considered suitable for a rapid maxillary expansion appliance is also dictated by

\section{Figure 2-Maxillary midline diastema develops} during expansion.

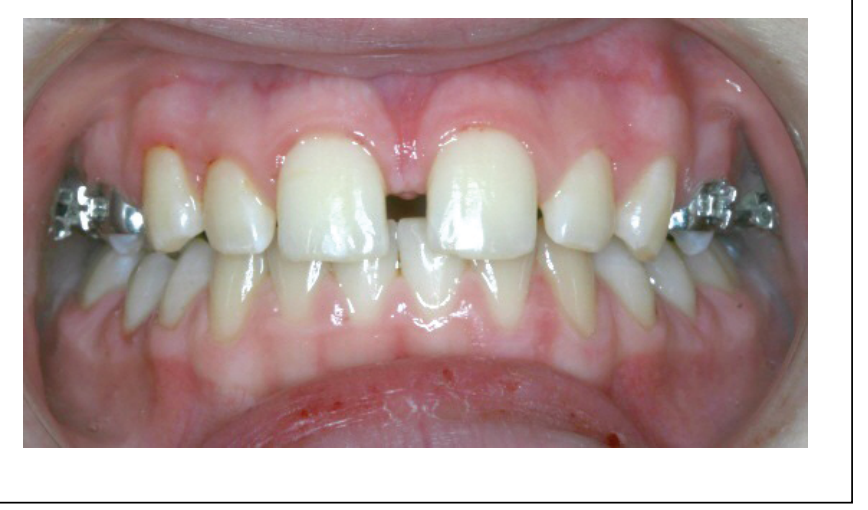

his or her ability to tolerate sizing and seating of bands, dental impressions, cementation of the lab-fabricated expansion appliance, and daily activation of the expansion screw. Treatment of an apprehensive or uneasy patient may need to be deferred until further maturity enables cooperation with the procedures.

The patient must also be able to accept the presence of a fixed bulky palatal appliance that interferes with eating and requires enhanced oral hygiene measures for many months.

\section{Appliance Management}

Responsibility for appliance effectiveness includes not only the provider but also the patient and the parent or caregiver to manage the following:

1. Intra-oral activation of the expansion screw

2. Appliance dislodgment

3. Retention of the correction after the active phase of expansion

4. Other complications

\section{Activation of the Expansion Screw}

After the appliance is fixed to the dentition, the parent or caregiver will need to activate the expansion screw by turning it once or twice a day according to the practitioner's recommendations. This necessitates cooperation and patience from the patient as the parent must identify intra-orally the hole of the expansion screw in which to insert the key in order to turn the screw.

\section{Appliance Dislodgment}

Dietary restrictions are advised to minimize dislodgment of the appliance from the dentition. "Oooey, gooey, sticky, chewy" foods should be avoided as should ice, popcorn, and other hard foods. Unfavorable crown anatomy may preclude adequate fitting of bands which may loosen even in the most compliant patient. Occasionally bands fracture, and a new appliance will need to be fabricated.

Usually the expander dislodges only partially, but it must be completely removed in order to repair and properly re-seat. If unable to be re-seated at the same appointment, loss of expansion will occur, and when the appliance is re-cemented at a subsequent appointment, it requires reversing the screw advancement to match appliance width to the maxillary width. 


\section{Retention Protocol}

As noted earlier, even after the suture has remineralized, dental and skeletal relapse in the expansion occurs. To avoid complete loss of the correction, retention is a critical aspect of the procedure.

The retention protocol should be discussed with the patient and parent. Protocols may include keeping the expander in place until comprehensive orthodontics is undertaken. If the expander is removed after 3-6 months, it may be replaced with another fixed but less bulky appliance called a transpalatal arch (Figure 4).

Removable retainers such as a Hawley retainer rely on excellent compliance from the patient and may be contraindicated in the mixed dentition as the retainer depends on close adaptation to dental units to maintain corrections. A thin clear plastic horseshoe retainer that covers only the dentition will likely have insufficient strength to prevent skeletal relapse.

Retention poses particular challenges in patients in the early or mixed dentition. Not only must retention be maintained for years, but also as teeth exfoliate, critical dental units that secure the skeletal expansion are lost. Careful follow-up is required to ensure that the retainer does not impede eruption of permanent teeth and to adapt or replace the retainer during the tooth exchange period.

\section{Other Complications}

Because expansion forces are applied to the teeth at a distance coronal to their center of rotation, teeth will tip buccally in addition to their lateral displacement as the palatal bones separate at the mid-palatal suture. The absence of suture release during expansion (e.g., due to suture maturation) will lead to excessive buccal crown tipping of the teeth attached to the expander and little to no skeletal expansion. This not only creates an unstable occlusion but is also prone to near complete relapse of the accomplished expansion.

The force required to move a tooth is very light and in the range of 1-4 oz. Even with such light forces, orthodontic treatment has been associated with root resorption. ${ }^{16}$ Forces of the magnitude applied with expansion appliances have been reported to range from 3 to $10 \mathrm{lbs},{ }^{17}$ but little is known about the risk or extent of root resorption secondary to these force levels. Nevertheless RME has been successfully used for decades in orthodontic treatment with no apparent untoward clinical sequelae of this nature, but use of this procedure in much younger patients bears careful monitoring to ensure that unfavorable side effects do not occur.

Little research has been conducted into examining risk of root fenestrations or dehiscences as the roots of teeth are displaced buccally toward the cortical plate, however it has been raised as a potential concern. ${ }^{18}$ In younger patients, the bone is less mature and mineralized ("softer"), leading to speculation that it might be a greater risk to undertake expansion in such patients. On the other hand, one may argue that less force is required to separate the midline palatal suture and/ or that the alveolar bone of younger patients is better able to adapt to expansion and may tolerate such forces better than mature alveolar bone. The studies are lacking.

Finally, non-compliance with or poor design of the retention protocol will result in loss of the expansion. While this will
Figure 3-“Unilateral” crossbite: symmetric maxillary crossbite with mandibular functional shift to patient's left to establish occlusal contacts.
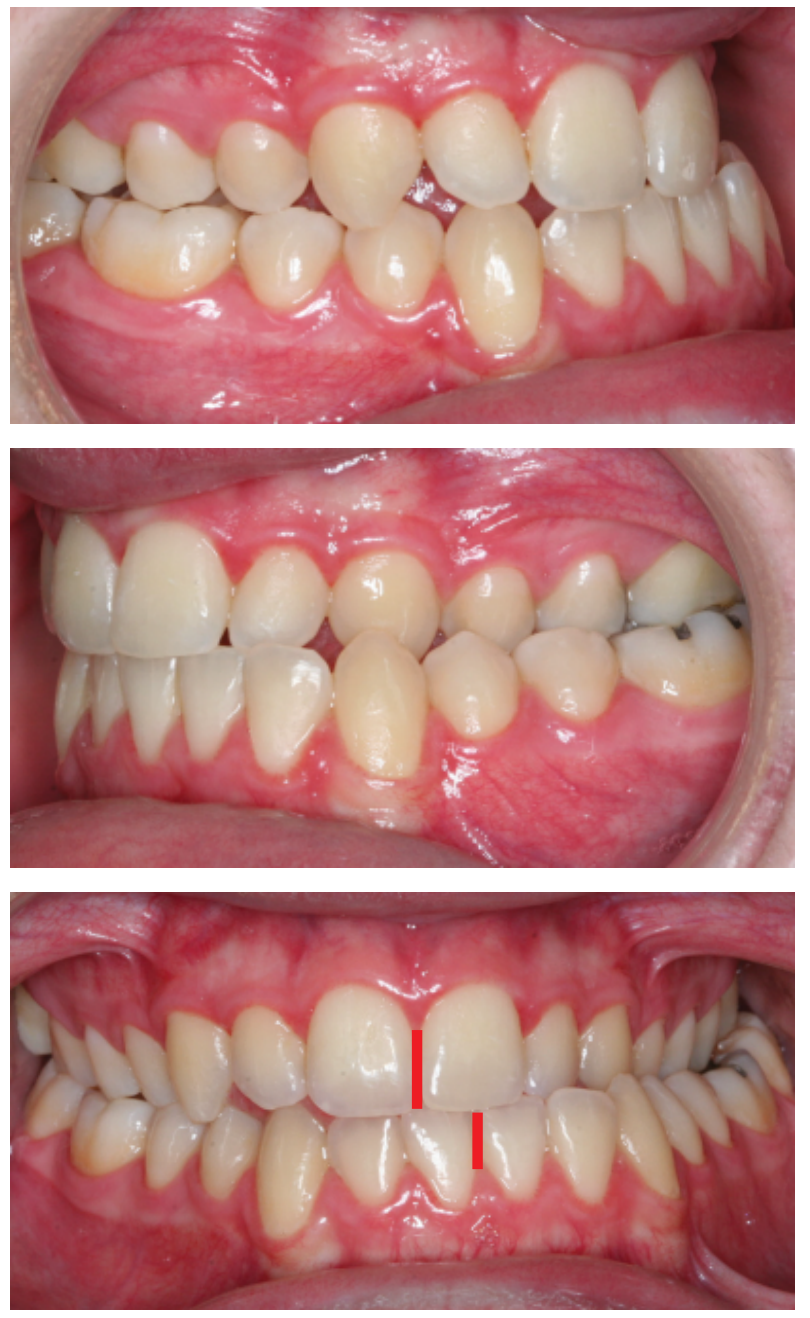

Non-coincident midlines are marked by vertical red lines in bottom image.

Figure 4-Transpalatal arch.

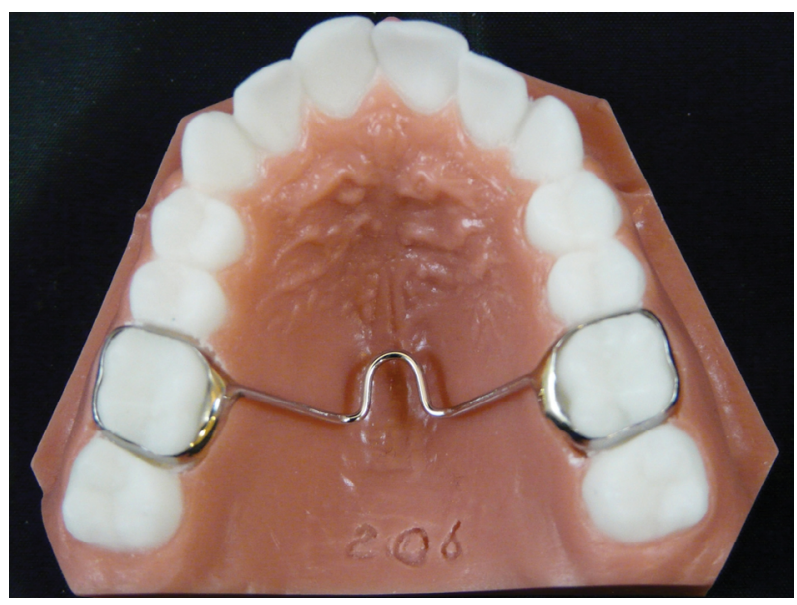

Image courtesy of John Mark Griffies, DDS 
certainly have implications for subsequent orthodontic treatment, it may have variable implications on the management of pediatric sleep disordered breathing. If the expansion is accomplished and maintained at a critical point in airway or craniofacial skeletal growth and development, it is possible that the airway benefit will have been attained and relapse of the crossbite may be inconsequential other than from an orthodontic perspective. Clearly more research is needed to guide decisions about timing of early intervention.

\section{Further Research}

While RME is a promising tool to manage SDB in pediatric patients, further investigation is required to elucidate:

1. The optimal age for initiating RME treatment

2. The feasibility of offering this treatment in patients prior to the eruption of permanent posterior teeth

3. The design and duration of effective retention protocols

4. The benefits/risks in patients without significant maxillary constriction

5. The long-term cognitive, behavioral, and health outcomes of early intervention with RME

Traditionally orthodontists proceed with RME treatment to correct posterior crossbites only after first molars have erupted and often defer until patients are close to puberty. Risks and benefits need to be clarified for treating sleep disordered breathing in children who may still be only in the primary dentition before recommending this orthodontic procedure as an early intervention. Carefully designed clinical studies are necessary to develop guidelines for use of rapid maxillary expansion as a viable treatment modality for pediatric patients diagnosed with or deemed at risk for pediatric sleep disordered breathing.

\section{CITATION}

Sheats RD. Rapid maxillary expansion for pediatric sleep disordered breathing. Journal of Dental Sleep Medicine 2014;1(3):131-134.

\section{REFERENCES}

1. Baydas B, Yavuz I, Uslu H, Dagsuyu I, Daylan I. Nonsurgical rapid maxillary effects on craniofacial structures in young adult females. A bone scintigraphy study. Angle Orthod 2006;76:759-67.

2. Leonard R, Sicurezza E, Cutrera A, Barbato E. Early post-treatment changes of circumaxillary sutures in young patients treated with rapid maxillary expansion. Angle Orthod 2011;81:36-41.
3. Bishara SE, Staley RN. Maxillary expansion: clinical implications. Am J Orthod Dentofacial Orthop 1987;91:3-14.

4. Ekstrom C, Henrikson CO, Jensen R. Mineralization in the midpalatal suture after orthodontic expansion. Am J Orthod 1977;71:449-55.

5. Geran RG, McNamara JA, Baccetti T, Franchi L, Shapiro LM. A prospective long-term study on the effects of rapid maxillary expansion in the early mixed dentition. Am J Orthod Dentofacial Orthop 2006:129:631-40.

6. Haas AJ. The treatment of maxillary deficiency by opening the midpalatal suture. Angle Orthod 1965;35:200-17.

7. Wertz RA. Changes in nasal airflow incident to rapid maxillary expansion. Angle Orthod 1968;38:1-11.

8. Hershey HG, Stewart BL, Warren DW. Changes in nasal airway resistance associated with rapid maxillary expansion. Am J Orthod 1976;69:274-84.

9. Timms DJ. The reduction of nasal airway resistance by rapid maxillary expansion and its effect on respiratory disease. J Laryngol Otol 1984;98:357-62.

10. Warren DW, Hershey G, Turvey T, Hinton VA, Hairfield WM. The nasal airway following maxillary expansion. Am J Orthod Dentofacial Orthop 1987;91:111-6.

11. Pirelli P, Saponara M, Guilleminault C. Rapid maxillary expansion in children with obstructive sleep apnea syndrome. Sleep 2004;27:761-6.

12. Villa MP, Malagola C, Pagani J, et al. Rapid maxillary expansion in children with obstructive apnea sleep syndrome: 12 -month follow-up. Sleep Med 2007;8:128-34.

13. Villa M P, Rizzoli A, Miano S, Malagola C. Efficacy of rapid maxillary expansion in children with obstructive sleep apnea syndrome: 36 months of follow-up. Sleep Breath 2011;15:179-84.

14. Persson M, Thilander B. Palatal suture closure in man from 15 to 35 years of age. Am J Orthod 1977;72:42-52.

15. Kraut RA. Surgically assisted rapid maxillary expansion by opening the midpalatal suture. J Oral Maxillofac Surg 1984;42:651-5.

16. Roberts-Harry D, Sandy J. Orthodontics. Part 11: Orthodontic tooth movement. Br Dent J 2004:196:391-4.

17. Isaacson RJ, Ingram AH. Forces produced by rapid maxillary expansion 2: Forces present during treatment. Angle Orthod 1964;34:261-9.

18. Davidovitch M, Efstathiou S, Sarne O, Vardimon A. Skeletal and dental response to rapid maxillary expansion with 2- versus 4-band appliances. J Am Orthod Dentofacial Orthop 2005;127:483-92.

\section{SUBMISSION \& CORRESPONDENCE INFORMATION}

Submitted for publication September, 2014 Accepted for publication September, 2014

Address correspondence to: Rose D. Sheats, University of North Carolina, School of Dentistry, CB 7450, Chapel Hill, NC 27599; E-mail: Rose_ Sheats@unc.edu

\section{DISCLOSURE STATEMENT}

This was not an industry supported study. The authors have indicated no financial conflicts of interest. 


\section{A Little Insurance Information That Might Keep You Out of Harm's Way}

David B. Schwartz, DDS, Diplomate, ABDSM

The Center for Sleep Medicine, Skokie, IL

$\mathrm{W}$

hile we are all familiar with or at least more aware of our dental malpractice insurance, we truly cross over into a different world when we are performing oral appliance therapy. One suggestion I can make is to contact your individual insurance company and have them provide you with a definitive coverage for treating snoring and sleep apnea. This may come in the form as an additional endorsement or simply as a letter from the underwriter stating that you are covered for providing this procedure when it is ordered by a physician. The underwriter is just trying to protect you and also the insurance provider. A simple phone call to your insurance representative can save you lots of aggravation down the road. Each, state and carrier will have different rules so check with your malpractice insurance provider to clarify anything that you may be unclear on.

\section{CITATION}

Schwartz DB. A little insurance information that might keep you out of harm's way. Journal of Dental Sleep Medicine 2014;1(3):135.

\section{SUBMISSION \& CORRESPONDENCE INFORMATION}

Submitted for publication September, 2014 Accepted for publication September, 2014

Address correspondence to: David B. Schwartz, 9933 Lawler Avenue, Suite 340, Skokie, IL 60077; Tel: (847) 677-2808; Fax: (847) 677-3214; E-mail: tzanerdoc@aol.com

\section{DISCLOSURE STATEMENT}

Dr. Schwartz has indicated no financial conflicts of interest. 



\section{What's in a Name?}

\section{Todd D. Morgan, DMD, Diplomate, ABDSM}

Scripps Memorial Hospital-Encinitas, Oral Medicine Section, Encinitas, CA
T am often struck by how the right word can make a difference. 1 Two words may mean essentially the same thing, but carry a different connotation depending on the context for each listener. For example, the word "titration," according to Wikipedia, means: "quantitative chemical analysis that is used to determine the unknown concentration of an identified analyte." In the context of Sleep Medicine, titration means: "to determine the required pressure to be set on a CPAP flow generator to abolish both apnea \& hypopnea events." So this leads to the question: Does the term titration accurately apply to what we do in oral appliance therapy when we adjust the mandible's horizontal and vertical positions to improve airway caliber?

When I began practicing dental sleep medicine in 1988, I was introduced to the word titration as it related to the graduated addition of air pressure to the patient upper airway at the CPAP mask interface. Back then, I would marvel at this technique and watch as the squiggly lines produced on paper by the PSG recorders were smoothed out with CPAP titration. This was "a good thing," according to Dr. Phillip Westbrook, a pioneer in sleep medicine.

Early in my practice I was using a single piece device now termed a "monoblock design." Before the George Gauge was available I made an educated guess about the protrusive starting point with a wax bite. I saw many awesome results and also failures. In the failures I suspected that I had missed determining the best protrusive position. The technique of cutting the arch trays apart and resetting forward allowed me to improve my results, and I had no compunction about doing this many times over for a patient. I performed my primitive alterations by cutting things apart and then re- uniting the pieces with acrylic in progressive jaw positions, all while using an oximeter to track progress. I'm not sure now when the term titration began to be applied to oral appliance adjustments.

I had been bitten too many times by patients who said they were doing fine only to find the follow up PSG results were lousy. Now there was no turning back-recalibrating the monoblocks while using home sleep testing put my destiny in my own hands. But was that titration?
The first adjustable appliance I fitted was the Klearway. After 10 years, no more tedium and smelling acrylic! The term titration appeared in the literature in the early 2000's and it didn't take long for practitioners to realize the advantages of adjustable devices. It was also evident that the humbling truth was that we couldn't help all of our patients, even with new technology, and our best efforts. Turn the page. Now the dentists are invading the sleep lab and finding ways to adjust appliances during the PSG-something that could not have been imagined at the beginning of this journey.

Now back to the word. I believe it is time for a more accurate description of what we do. In searching for the best terminology to replace "titration" I keep arriving at the term "calibration." Indeed, this is a much better description of what we do.

So here's to continued growth and change within our field, including the nomenclature! My humble suggestion for change is to endorse a term that more accurately describes what we do with oral appliances. The term calibration fits and it is one that we can own.

\section{CITATION}

Morgan TD. What's in a name? Journal of Dental Sleep Medicine 2014;1(3):137.

\section{SUBMISSION \& CORRESPONDENCE INFORMATION}

Submitted for publication August, 2014

Accepted for publication September, 2014

Address correspondence to: Todd Morgan, DMD 320 Santa Fe Drive Suite105 Encinitas, CA 92024; Tel: (760) 436-9292; Fax: (760) 436-9332;

E-mail: todd@toddmorgan.com

\section{DISCLOSURE STATEMENT}

This was not an industry supported study. Dr. Morgan has indicated no financial conflicts of interest. 



\title{
Generating Awareness for Oral Appliance Therapy
}

\author{
L.C. Williams \& Associates, Public Relations Agency of Record for AADSM since 2012
}

Chicago, IL

$\mathrm{D}$ iagnosis of sleep apnea is growing year-over-year, driving more patients to seek treatment options, and making it more vital than ever before to increase awareness of oral appliance therapy as an effective treatment alternative.

In support of its membership and the field of dental sleep medicine, the American Academy of Dental Sleep Medicine is undertaking a multi-year public relations initiative to educate the public about oral appliance therapy and drive patients who can't tolerate CPAP to LocalSleepDentist.com to find local AADSM members. This campaign has reached people with national media stories, industry trade articles and social media events, generating more than 175 million impressions in two years-with more on the way.

\section{LAYING THE FOUNDATION FOR OAT}

\section{Educating Patients}

Since 2012, the AADSM public relations campaign has gotten stories about OAT featured in a broad range of national consumer media outlets, including key men's, women's and health magazines, health-focused radio programs and websites, news broadcasts and newspapers in the U.S. and Canada. As a result, oral appliance therapy has been national media stories, including:

- First for Women profiled an AADSM member and his patient in a story about upper airway resistance syndrome in its February 2013 issue, which was distributed to more than 1.2 million readers. The two-page article explains how oral appliance therapy is an effective, "life-changing" solution for people suffering from the disease and paints a very positive picture of the solutions dental sleep medicine can provide.

- Real Simple, a national women's lifestyle magazine, featured oral appliance therapy as a remedy for snoring in the April 2013 edition, reaching more than 1.9 million readers. The article, "Snoring Remedies", informs consumers that OAT is a comfortable, dentist-provided solution with an 80 percent success rate for snorers and patients with mild to moderate sleep apnea.

- WomansDay.com and Prevention.com, websites for the national magazines of the same names, both included OAT in stories about sleep apnea and snoring. The March 2013 Woman's Day article focused on the potential health effects and treatment options for snoring with a tipsfocused story. The March 2014 Prevention article centered on surprising causes for erectile dysfunction, citing sleep apnea as a trigger for the problem and OAT as a possible solution that's easier to sleep with.

- Huffington Post, a wide-reaching news website, featured Dr. B Gail Demko, AADSM past president, as a guest expert blogger. Her blog post on "Unmasking Sleep Apnea
Treatments: What to Try If CPAP Doesn't Work for You" appeared in May 2014 reaching more than three million potential readers.

- USA TODAY insert on lung health was co-sponsored by both AADSM and AASM. The features included a Q\&A with AADSM founding president Dr. Robert Rogers and an advertisement driving sleep apnea sufferers to find a local AADSM member. The supplement reached more than 1.4 million readers.

- Television segments on nationally-syndicated morning news programs, including "The Daily Buzz" and "Life Love Shopping," aired in February 2013 and August 2013. In total, the segments ran in more than 200 markets nationwide, reaching nearly three million viewers. The segments highlighted oral appliance therapy as an effective alternative to CPAP and an easier travel solution for sleep apnea sufferers on the go, and featured the LocalSleepDentist.com website.

\section{Social Media Builds Buzz}

Beyond traditional media outreach, the AADSM PR campaign also reaches potential patients through partnerships with a number of high-profile bloggers. Social efforts have included:

- A "Snoring Mad" online chat, held on Twitter in October 2013, drove conversations about sleep-related breathing issues and highlighted the solutions AADSM members can provide through the use of OAT. The chat had hundreds of participants, resulting in 2,500 tweets and more than 11 million online impressions.

- "Keep Calm and Sleep On” Blogger Tour, conducted November 2013-January 2014, tapped influential bloggers to educate consumers about OAT. Sixteen total blog posts included images of an oral appliance, information on the path to treatment and a call-to-action to visit localsleepdentist.com to find an AADSM dentist locally. The effort generated 1.1 million impressions and 1,700 reader comments including remarks "I need this. I really did not want to be hooked to a machine, so this would be great!" and "I think I might have sleep apnea, and I keep putting off asking my doctor about it. After reading the list of scary health problems, I will for sure check into it!”

\section{Reaching Industry Partners}

To advance dental sleep medicine among sleep doctors and dentists, AADSM reached out to industry journals and magazines to help spur the continued growth of the field. Through announcing facility accreditations, highlighting recent research studies and providing industry-leading bylined articles and webinar participation, AADSM has furthered awareness of dental sleep medicine in the medical community. 
Over the past two years, AADSM experts have been profiled in leading industry publications including AGD Impact, New Dentist, Sleep Review, DentistyIQ, ADVANCE for Respiratory Care \& Sleep Medicine and more.

\section{EMPOWERING MEMBERS}

In addition to telling the story about OAT to national media, the AADSM is working to provide individual members with the tools they need to replicate this PR success at the local level by providing the AADSM Member PR Toolkit. The Toolkit is filled with customizable press releases, phone scripts, media outreach tips and advice that allow members to leverage the national campaign at the local level. All of the pieces can be used to establish relationships with influential media and sleep physicians-and ultimately generate phone calls and business for AADSM members. Download the updated AADSM Member PR Toolkit today at http://www.aadsm.org/ PromotionalResources.aspx, and build buzz for oral appliance therapy in your community!

\section{FALL PR ACTIVITIES}

The AADSM continues to generate awareness with prospective patients in the third year of its public relations initiative.

- In September, the AADSM coordinated publicity for oral appliance therapy in a series of television segments about fall wellness. The segments aired on 10 local morning television news programs as well as nationally on "The Daily Buzz."

- In October, AADSM Board President Kathleen Bennett and board member Thomas Schell traveled to New York City on behalf of AADSM to introduce influential health reporters from top magazines to oral appliance therapy and explain the benefits of the treatment.
- In early 2015, the AADSM will conduct a proprietary survey about the effects of sleep apnea on bed partners. The resulting statistics will be used to build awareness of the need for an effective treatment with which patients can more easily comply.

Building awareness for oral appliance therapy among prospective patients, new dentists and sleep physicians is a crucial step towards the continued growth of dental sleep medicine. With AADSM support and ongoing, impactful media outreach, a conversation about alternative sleep apnea treatments is beginning - and generating awareness across the country.

\section{AADSM MEMBER PUBLIC RELATIONS TOOLKIT}

\section{Activation Tip}

Put the toolkit to work for you today by customizing the "New Year's Resolution Press Release" to your practice. This seasonal press release raises awareness about the relationship between getting quality sleep and achieving health resolutions, and positions a trip to the dentist as a necessary next step to a healthier New Year. To use the release, simply download the toolkit from the Promotional Resources page of the AAADSM website and replace the red text in the release Word document with information specific to your practice. Make sure all of the text is turned back to black before you email it to health reporters at local newspapers. Reporter email addresses can usually be found on the newspaper's website. This release should be sent to reporters in early December to get a jump on holiday vacations, with a short note offering more information and an interview with a local patient who used the treatment to better their sleep in 2014. 


\section{AADSM News and Updates}

AADSM Staff

AADSM National Office, Darien, IL

\section{AADSM EDUCATIONAL CALENDAR OF EVENTS}

2014

September 27: Practical Demonstration Course (Darien, IL - AADSM National Office)

October 9-14: ADA Annual Meeting (San Antonio, TX) AADSM sponsoring and presenting an educational program on dental sleep medicine

October 18-19:

- Advanced Dental Sleep Medicine Course (Washington, D.C.)

- Essentials of Dental Sleep Medicine Course (Washington, D.C.)

- Practice Management Course (half-day add-on) (Washington, D.C.)

December 6: Practical Demonstration Course (Darien, IL - AADSM National Office)

2015

February 28-March 1: Essentials of Dental Sleep Medicine Course (New Orleans, LA)

March 21: Practical Demonstration Course (Darien, IL - AADSM National Office)

TBD: Board Review Course

June 4-6: AADSM $24^{\text {th }}$ Annual Meeting (Seattle, WA)

\section{AADSM COMMITTEES UPDATE}

\section{Accreditation Committee}

The Accreditation Committee continues to review applications for Dental Sleep Medicine Facility Accreditation and is pleased to have seen 39 facilities become accredited to date. Accreditation solidifies dental sleep medicine professionals as instrumental to the sleep medicine team and helps facilities build strong medical partnerships. The committee looks forward to receiving your application, which is available for download at http://www.aadsm.org/Application.aspx.

\section{Annual Meeting Committee}

The Annual Meeting Committee is working diligently to finalize the program for the AADSM $24^{\text {th }}$ Annual Meeting that will be held Thurs., June 4-Sat., June 6, 2015 in Seattle, WA. The committee is also accepting session proposals and challenging case submissions for the 2015 Annual Meeting until December 1,2014 . This is your chance to influence the meeting's education program! Learn more about the session guidelines and how to submit materials by visiting http://aadsm.org/meetingabstracts. aspx.

\section{Education Committee}

The Education Committee is currently hosting its fall Study Club Program, which is designed for dentists new to dental sleep medicine. The committee is simultaneously preparing for the 2015 Study Club Program, which will be conducted via web conference to make the sessions more interactive. New educational offerings, such as live Q\&A sessions and online learning modules, will give AADSM members additional opportunities to learn more about dental sleep medicine from the convenience and comfort of their home or practice. Look for an announcement in your monthly NewsFlash email regarding the release dates of these exciting new educational opportunities!

\section{Health Policy Education Committee}

Recently, the Health Policy Education Committee developed frequently asked questions that provide members with answers to common questions about reimbursement for oral appliance therapy services. Members are encouraged to check out the FAQs on the AADSM website at http://www.aadsm.org/ managementresources.aspx. The committee is currently developing an online learning module to further educate members in areas of health policy.

\section{Research Committee}

The Research Committee is currently accepting abstract submissions for the 2015 Annual Meeting. To view the Call for Abstracts, please visit http://aadsm.org/meetingabstracts. aspx. The Research Committee looks forward to receiving your abstract submission by December 17, 2014. 



\section{Biographical Information for the 2014-2015 American Academy of Dental Sleep Medicine Board of Directors}

Leslie C. Dort, DDS, Diplomate, ABDSM, Editor-in-Chief Journal of Dental Sleep Medicine

Calgary, Alberta, Canada

\section{Kathleen M. Bennett, DDS, Diplomate, ABDSM}

President AADSM

Graduated SUNY Buffalo Dental School, 1983. GPR Buffalo General Hospital. Private Practice in general dentistry until 2005, Buffalo NY. Practices Dental Sleep Medicine exclusively, private practice in Cincinnati OH. Credentialed UC Health in Department of Internal Medicine UC West Chester Hospital Pulmonary Division of Sleep Medicine in January 2014. Previous positions: Director AADSM; Director ABDSM; and Chair, Annual Meeting Education committee member.

\section{Harold A. Smith, DDS, Diplomate, ABDSM}

President-Elect of AADSM

Fellow of American College of Dentists. Full time private practice, Indianapolis, IN. Practicing DSM since 1993. Dr. Smith's dental practice is into the 3rd generation of many of his patient's families.

\section{B. Gail Demko, DMD, Diplomate, ABDSM Immediate Past President of the AADSM}

Private practice in Weston, MA. Has been an active volunteer with the AADSM since 1998. Liason to the education committee and recipient of the Distinguished Service Award. Dr. Demko is the expert advisor to the FDA in the area of oral devices for the treatment of OSA.

\section{Leslie Dort, DDS, MSc (Health Res), Diplomate, ABDSM}

AADSM Secretary/Treasurer

Private practice in Calgary, Alberta, Canada. General dentist since 1980, has limited her practice primarily to dental sleep medicine. Editor-in-Chief, Journal of Dental Sleep Medicine. Previous positions: AADSM BOD member at large; Chair, Annual Meeting Committee; and Chair, Education Committee.

\section{Steven C. Scherr, DDS, Diplomate, ABDSM}

AADSM Director

President-elect ABDSM. Private practice general dentist with focus on dental sleep medicine in Pikesville, MD. DSM Local Program Director for Johns Hopkins Medical School Sleep Fellowship Program. Previous positions: Chaired Task Force that developed Accreditation Standards; Co-chaired Oral Appliance Definition Consensus Conference; and Chair, Reimbursement Committee.
Rose Sheats, DMD, MPH, Diplomate, American Board of Orthodontics

AADSM Director

Retired in 2013 from full time academics in the University of North Carolina School of Dentistry Department of Orthodontics. Curently limits practice to dental sleep medicine at the UNC School of Dentistry in Chapel Hill, NC. Has been providing OAT since 1997. Associate Editor, Journal of Dental Sleep Medicine. Previous positions: Chair, Education Committee and Chair, Advanced Course at annual meeting

\section{Thomas G. Schell, DMD, Diplomate, ABDSM} AADSM Director

General private practice in New Hampshire since 1993. Research Committee member. Adjunctive Faculty of Geisel School of Medicine at Dartmouth College, Hanover, New Hampshire. Oral Appliance Consensus conference member.

\section{David Schwartz, DDS, Diplomate, ABDSM AADSM Director}

General dentist since 1988 with a concentration in Dental Sleep Medicine since 1994. Practices part time at The Center for Sleep Medicine in Chicago area and has private practice in Skokie, IL. Previous positions: Chair, Advanced Course; Vice Chair, Advanced course; and Lecturer, Board Review Course and Mini Board Review Course.

\section{Grant D. Hensley, DDS}

AADSM Director

Graduated from University of Tennessee in 1987 and practiced in the US Army for six years prior to entering private practice in Tennessee. Began working with OSA patients in general dentistry practice 15 years ago.

\section{Kevin Postol, DDS, Diplomate, ABDSM AADSM Director}

General private practice since 1992. Speaker for the Essentials of Dental Sleep Medicine Course. Previous Positions: Health Education Committee and Chair of the Essentials of Dental Sleep Medicine Course. 



\title{
Membership Survey Summary
}

\author{
Leslie C. Dort, DDS, Diplomate, ABDSM, Editor-in-Chief Journal of Dental Sleep Medicine
}

Calgary, Alberta, Canada

The AADSM recently engaged McKinley Advisors, Chicago, 1 Illinois, to conduct a membership survey. The objectives of the survey were to:

- Gain a better understanding of the current needs and expectations of its members

- Identify areas where the organization is performing well as well as gaps to address

- Discover opportunities to increase value and satisfaction for members

The survey involved telephone interviews, an electronic survey, analyses of results, detailed cross-tabulation and thorough review of the open-ended questions. Over a period of 15 days (February 28-March 14, 2014) 20 telephone interviews were conducted and surveys were sent to 2,948 email addresses. Complete responses were received from 375 members and partial responses from 59 members.

Respondents identified themselves primarily as dentists $(90 \%)$. Dental professors comprised $2 \%$ of respondents and $1 \%$ were dental researchers. Most respondents (68\%) spent less than $25 \%$ of their time practicing dental sleep medicine.

The satisfaction of members regarding value of member benefits compared to dues placed the AADSM in the top one third of 45 comparable professional and trade associations. In addition to asking members what they valued most about AADSM, the survey asked them to provide thoughts on how the Academy could provide stronger value. Responses were again analyzed and grouped according to common theme. Members were most interested in more education and hands-on programs as well as issues related to professional standards and stronger integration with the medical community.

\section{KEY FINDINGS}

\section{Education}

Education is central to the AADSM membership value proposition. Access to education is a primary reason why members join AADSM, and why they continue membership.

\section{Marketing}

Members want AADSM to engage more in marketing and public outreach efforts and feel that AADSM should take on a greater role in educating members on effective ways to market and promote their own practice.

\section{Integration}

Overall, members would like to see stronger integration between the medical and dental community. Specific resources were mentioned, such as the referral database, that cultivates this integration. Members spoke of the need for creating and defining more policies and procedures as a catalyst for stronger integration. Open-ended responses from members exhibited a preference for collaboration and integration between dentists and physicians as opposed to autonomy.

\section{Recruitment}

Most members joined AADSM because of a colleague or peer. Professional development and the Annual Meeting were also among the top reasons for joining.

\section{Insurance}

Issues understanding insurance policies and reimbursement permeates the entire AADSM membership, and especially the younger, less tenured demographic.

\section{Advocacy}

Increased advocacy efforts on behalf of the profession continue to play an extremely important role in member value. In fact, $80 \%$ of members ranked it as a major decision factor in joining AADSM.

\section{Engagement}

The e-news letter, Annual Meeting and continuing education are the top platforms for engagement. Nearly $70 \%$ of respondents said they read the e-news updates, while $63 \%$ said they have attended the AADSM Annual Meeting and 51\% said they have taken advantage of CE opportunities.

The survey has provided a wealth of information that the AADSM leadership will use to build value and satisfaction for members in the future. All members are encouraged to continue to provide feedback and suggestions. 
